\title{
Highly Efficient Synthesis of Isoquinolines via Nickel-Catalyzed Annulation of 2-Iodobenzaldimines with Alkynes; Evidence for Dual Pathways of Alkyne Insertion
}

\author{
Korivi Rajendra Prasad, Chien-Hong Cheng* \\ Department of Chemistry, National Tsing Hua University,Hsinchu, Taiwan 30013 \\ chcheng@mx.nthu.edu.tw
}

\section{Supporting Information}

Table of Contents

1. General procedure of synthesis $\quad$ S2.

2. Spectral data of the compounds S3-S8.

3. NOE data for the selected compounds $\quad$ S8.

4. ${ }^{1} \mathrm{H}$ and ${ }^{13} \mathrm{C}$ NMR spectra of selected compounds $\quad \mathrm{S} 10-\mathrm{S} 45$. 
General Procedure for the Preparation of Isoquinolines: A round-bottom side-arm flask $(25 \mathrm{~mL})$ containing $\mathrm{NiBr}_{2}(\mathrm{dppe})(3.1 \mathrm{mg}, 0.0050 \mathrm{mmol})$ and zinc powder (13 mg, $0.20 \mathrm{mmol}$ ) was evacuated and purged with nitrogen gas three times. Freshly distilled acetonitrile $(3.0 \mathrm{~mL}), 2$-iodobezaldimine $(0.10 \mathrm{mmol})$ and alkyne $(0.10 \mathrm{mmol})$ were added to the system and the reaction mixture was stirred at $80{ }^{\circ} \mathrm{C}$ for the desired time (20 min or $3 \mathrm{~h}$ ). The reaction mixture was cooled to room temperature and diluted with dichloromethane. The mixture was filtered through a silica gel pad and washed thoroughly with dichloromethane. The filtrate was concentrated in a rotary evaporator and the residue was separated on a silica gel column using a mixture of hexanes and ethyl acetate as eluent to afford the desired pure product 3. Compounds 3a-p were synthesized according to this procedure and the spectral data of these compounds are as follows. The spectral data of products $3 a,{ }^{1} 3 \mathbf{3 c},{ }^{3} \mathbf{3 d},{ }^{2} \mathbf{3 f}, 3 \mathbf{3 l},{ }^{4} 3 \mathbf{3},{ }^{3} \mathbf{3 p}^{5}$ and $3 \mathbf{q}^{6}$ were reported in the literature.

Table. Results for the Formation of $\mathbf{3 a}$ in the Presence of Various Catalysts and solvents ${ }^{a}$

\begin{tabular}{cccccc}
\hline entry & catalyst & yield & entry & catalyst & yield \\
\hline 1 & $\mathrm{NiBr}_{2}\left(\mathrm{PPh}_{3}\right)_{2}, \mathrm{Zn}$ & 91 & 7 & $\mathrm{NiBr}_{2}(\mathrm{dppe}), \mathrm{THF}$ & 63 \\
2 & $\mathrm{NiBr}_{2}(\mathrm{dppe}), \mathrm{Zn}$ & 98 & 8 & $\mathrm{NiBr}_{2}(\mathrm{dppe})$ & 0 \\
3 & $\mathrm{NiCl}_{2}\left(\mathrm{PPh}_{3}\right)_{2}, \mathrm{Zn}$ & 80 & 9 & $\mathrm{Zn}$ & 0 \\
4 & $\mathrm{Ni}(\mathrm{COD})_{2}+\mathrm{P}(\mathrm{o}-\mathrm{tol})_{3}$ & $>99$ & 10 & $\mathrm{CoI}_{2}(\mathrm{dppe}), \mathrm{dppe}$ & 0 \\
5 & $\mathrm{NiBr}_{2}(\mathrm{dppe})$ & 98 & 11 & $\mathrm{CoCl}_{2}(\mathrm{dppe}), \mathrm{dppe}$ & 0 \\
6 & $\mathrm{NiBr}_{2}(\mathrm{dppe}), \mathrm{DMF}$ & 94 & & &
\end{tabular}

${ }^{a}$ All the reaction were carried out in nitrogen atmosphere using 1a, $(0.10 \mathrm{mmol})$, alkyne 2a $(0.10$ mmol), metal complex $(0.0050 \mathrm{mmol})$ and $\mathrm{Zn}(0.30 \mathrm{mmol})$ in $\mathrm{CH}_{3} \mathrm{CN}$ or the solvent mentioned in each entry $(3.0 \mathrm{~mL})$ at $80^{\circ} \mathrm{C}$ for $20 \mathrm{~min}$. 1a and $2 \mathbf{a}$ as reactants at $80^{\circ} \mathrm{C}$ for $20 \mathrm{~min}$. All the yields mentioned above are NMR yields using mesitylene as internal standard. 
3,4-Diphenylisoquinoline (3a): White solid; $\mathrm{mp}=170{ }^{\circ} \mathrm{C} ; \mathrm{R}_{\mathrm{f}}=0.41(15 \%$ ethyl acetate in hexanes); ${ }^{1} \mathrm{H}$ NMR $\delta$ 7.17-7.21 (m, 3H), 7.23-7.25 (m, 2H), 7.33-7.37 (m, 5H), 7.59-7.61 (m, 2H), $7.67(\mathrm{dd}, J=6.0$ and $3.0 \mathrm{~Hz}, 1 \mathrm{H}), 8.04(\mathrm{dd}, J=6.5$ and $3.0 \mathrm{~Hz}, 1 \mathrm{H})$ and $9.37(\mathrm{~s}, 1 \mathrm{H}) ;{ }^{13} \mathrm{C}$ NMR $\delta 125.6,126.9,127.1,127.3,127.6,127.6,128.3,130.2$, 130.5, 130.7, 131.2, 135.9, 137.2, 140.6, 150.5 and 151.7; $\mathrm{HRMS}\left(\mathrm{EI}^{+}\right) 281.1200$ (cal for $\mathrm{C}_{21} \mathrm{H}_{15} \mathrm{~N}$ 281.1204); IR 1446, 1497, 1614 and $3058 \mathrm{~cm}^{-1}$.

3,4-Diethylisoquinoline (3b): Viscous liquid; $\mathrm{R}_{\mathrm{f}}=0.39$ (15\% ethyl acetate in hexanes); ${ }^{1} \mathrm{H}$ NMR $\delta 1.29$ (t, $\left.J=7.5 \mathrm{~Hz}, 3 \mathrm{H}\right), 1.37$ (t, $\left.J=7.5 \mathrm{~Hz}, 3 \mathrm{H}\right), 3.01(\mathrm{q}, J=7.5$ Hz, 2H), 3.07 (q, $J=7.5 \mathrm{~Hz}, 2 \mathrm{H}), 7.56(\mathrm{t}, J=7.5 \mathrm{~Hz}, 1 \mathrm{H}), 7.74(\mathrm{t}, J=7.8 \mathrm{~Hz}, 1 \mathrm{H}), 7.96$ $(\mathrm{d}, J=8.0 \mathrm{~Hz}, 1 \mathrm{H}), 8.01(\mathrm{~d}, J=8.5 \mathrm{~Hz}, 1 \mathrm{H})$ and $9.12(\mathrm{~s}, 1 \mathrm{H}) ;{ }^{13} \mathrm{C}$ NMR $\delta$ 14.7, 15.0, $20.8,27.4,123.0,126.5,127.0,128.8,130.6,131.3,135.8,148.8$ and 152.0; $\mathrm{HRMS}\left(\mathrm{EI}^{+}\right)$ 185.1203 (cal for $\mathrm{C}_{13} \mathrm{H}_{15} \mathrm{~N}$ 185.1205); IR 1453, 1576, 1621, and $2967 \mathrm{~cm}^{-1}$.

4-Ethyl-3-phenylisoquinoline (3c): White solid; $\mathrm{mp}=103{ }^{\circ} \mathrm{C} ; \mathrm{R}_{\mathrm{f}}=0.36(15 \%$ ethyl acetate in hexanes ); ${ }^{1} \mathrm{H}$ NMR $\delta 1.28$ (t, $J=7.8 \mathrm{~Hz}, 3 \mathrm{H}$ ), 3.05 (q, $J=14.5$ and $7.8 \mathrm{~Hz}$, 2H), $7.41(\mathrm{dt}, J=7.0$ and $1.3 \mathrm{~Hz}, 1 \mathrm{H}), 7.46(\mathrm{t}, J=7.3 \mathrm{~Hz}, 2 \mathrm{H}), 7.52(\mathrm{~d}, J=7.5 \mathrm{~Hz}, 2 \mathrm{H})$, $7.60(\mathrm{t}, J=8.0 \mathrm{~Hz}, 1 \mathrm{H}), 7.75(\mathrm{t}, J=7.5 \mathrm{~Hz}, 1 \mathrm{H}), 8.00(\mathrm{~d}, J=8.0 \mathrm{~Hz}, 1 \mathrm{H}), 8.08(\mathrm{~d}, J=8.5$ $\mathrm{Hz}, 1 \mathrm{H})$ and $9.18(\mathrm{~s}, 1 \mathrm{H}) ;{ }^{13} \mathrm{C}$ NMR $\delta 15.6,21.8,123.6,126.6,127.6,127.7,127.8$, $128.2,128.4,128.6,129.2,130.1,130.5,135.2,141.3,150.0$ and 151.6; $\mathrm{HRMS}\left(\mathrm{EI}^{+}\right)$ 232.1136 (cal for $\mathrm{C}_{17} \mathrm{H}_{13} \mathrm{~N} 233.1204$ ); IR 1248, 1447, 1497, 1573, 1617 and $2976 \mathrm{~cm}^{-1}$.

Ethyl 3-phenylisoquinoline-4-carboxylate (3d): Viscous liquid; $\mathrm{R}_{\mathrm{f}}=0.38(15 \%$ ethyl acetate in hexanes); ${ }^{1} \mathrm{H}$ NMR $\delta_{\mathrm{H}}=1.03(\mathrm{t}, J=7.2 \mathrm{~Hz}, 3 \mathrm{H}), 4.23(\mathrm{q}, J=7.2 \mathrm{~Hz}, 2 \mathrm{H})$, $7.44(\mathrm{~m}, 3 \mathrm{H}), 7.64(\mathrm{dt}, J=7.8$ and $2.4 \mathrm{~Hz}, 1 \mathrm{H}), 7.7(\mathrm{td}, J=6.4$ and $2.2 \mathrm{~Hz}, 2 \mathrm{H}), 7.78(\mathrm{dt}$, $J=8.0$ and $2.0 \mathrm{~Hz}, 1 \mathrm{H}), 8.03(\mathrm{~d}, J=9.2 \mathrm{~Hz}, 2 \mathrm{H})$ and $9.36(\mathrm{~s}, 1 \mathrm{H}) ;{ }^{13} \mathrm{C} \mathrm{NMR} \delta 13.6,61.8$, 
$123.4,124.1,126.8,127.6,128.0,128.4,128.6,128.8,131.8,133.4,140.0,150.9,153.4$ and 168.6; HRMS (EI') 277.1100 (cal for $\mathrm{C}_{18} \mathrm{H}_{15} \mathrm{NO}_{2}$ 277.1103); IR 1221, 1498, 1564, 1574,1719 and $2981 \mathrm{~cm}^{-1}$.

1-(3-Phenylisoquilin-4-yl)ethanone (3e): White solid; $\mathrm{mp}=109{ }^{\circ} \mathrm{C} ; \mathrm{R}_{\mathrm{f}}=0.31$ (15\% ethyl acetate in hexanes); ${ }^{1} \mathrm{H}$ NMR $\delta 2.13(\mathrm{~s}, 3 \mathrm{H}), 7.47$ (m, 3H), $7.64(\mathrm{t}, J=7.8 \mathrm{~Hz}$, $1 \mathrm{H}), 7.68(\mathrm{dd}, J=7.8$ and $1.3 \mathrm{~Hz}, 2 \mathrm{H}), 7.75(\mathrm{t}, J=7.5 \mathrm{~Hz}, 1 \mathrm{H}), 7.90(\mathrm{~d}, J=8.0 \mathrm{~Hz}, 1 \mathrm{H})$, $8.04(\mathrm{~d}, J=8.0 \mathrm{~Hz}, 1 \mathrm{H})$ and $9.35(\mathrm{~s}, 1 \mathrm{H}) ;{ }^{13} \mathrm{C} \mathrm{NMR} \delta 32.4,123.9,127.1,127.6,128.1$, $128.8,129.1,129.5,131.8,132.4,139.6,149.2,153.3$ and 205.8; HRMS $\left(\mathrm{EI}^{+}\right) 247.0999$ (cal for $\mathrm{C}_{17} \mathrm{H}_{13} \mathrm{NO} 247.0997$ ); IR 1193, 1495, 1559, 1573, 1690 and $3058 \mathrm{~cm}^{-1}$.

(3-Phenylisoquinolin-4-yl)methanol (3f): White solid; $\mathrm{mp}=129{ }^{\circ} \mathrm{C} ; \mathrm{R}_{\mathrm{f}}=0.26$ ( $30 \%$ ethyl acetate in hexanes ); ${ }^{1} \mathrm{HNMR} \delta 5.08(\mathrm{~s}, 2 \mathrm{H}), 7.43(\mathrm{~m}, 1 \mathrm{H}), 7.48(\mathrm{t}, J=7.5 \mathrm{~Hz}$, 2H), $7.66(\mathrm{~m}, 3 \mathrm{H}), 7.82(\mathrm{t}, J=7.8 \mathrm{~Hz}, 1 \mathrm{H}), 8.03(\mathrm{~d}, J=7.8 \mathrm{~Hz}, 1 \mathrm{H}), 8.30(\mathrm{~d}, J=8.4 \mathrm{~Hz}$, $1 \mathrm{H})$ and $9.28(\mathrm{~s}, 1 \mathrm{H}) ;{ }^{13} \mathrm{C}$ NMR $\delta 59.4,123.9,125.9,127.2,127.7,128.3,128.3,128.3$, 129.7, 131.4, 136.0, 139.7, 152.2 and 152.5; $\mathrm{HRMS}\left(\mathrm{EI}^{+}\right) 235.0994$ (cal for $\mathrm{C}_{15} \mathrm{H}_{11} \mathrm{~N}$ 235.0997); IR 1261, 1447, 1575, 1621, 1714 and $2963 \mathrm{~cm}^{-1}$.

4-(Trimethylsilyl)-3-phenylisoquinoline(3g): White solid; $m p=161{ }^{\circ} \mathrm{C} ; \mathrm{R}_{\mathrm{f}}=$ 0.33 (10\% ethyl acetate in hexanes); ${ }^{1} \mathrm{H}$ NMR $\delta 0.13$ (s, 9H), 7.40-7.44 (m, 3H), 7.56 (dd, $J=7.8$ and $1.8 \mathrm{~Hz}, 2 \mathrm{H}), 7.58(\mathrm{dt}, J=7.8$ and $1.0 \mathrm{~Hz}, 1 \mathrm{H}), 7.71(\mathrm{dt}, J=7.8$ and $1.1 \mathrm{~Hz}$, 1H), $7.98(\mathrm{~d}, J=8.0 \mathrm{~Hz}, 1 \mathrm{H}), 8.16(\mathrm{~d}, J=8.5 \mathrm{~Hz}, 1 \mathrm{H})$ and $9.22(\mathrm{~s}, 1 \mathrm{H}) ;{ }^{13} \mathrm{C}$ NMR $\delta 2.2$, $126.3,126.7,127.1,127.7,128.0,128.2,128.6,129.7,130.1,141.0,144.4,152.3$ and 160.1; HRMS (EI $\left.{ }^{+}\right) 277.1287$ (cal for $\mathrm{C}_{18} \mathrm{H}_{19} \mathrm{NSi} 277.1287$ ); IR 1397, 1542, 1570, 1715 and $2953 \mathrm{~cm}^{-1}$. 
1-(3-Ethylisoquinolin-4-yl)ethanone (3h): Viscous liquid; $\mathrm{R}_{\mathrm{f}}=0.25$ (15\% ethyl acetate in hexanes); ${ }^{1} \mathrm{H}$ NMR $\delta 1.36(\mathrm{t}, J=7.8 \mathrm{~Hz}, 3 \mathrm{H}), 2.65(\mathrm{~s}, 3 \mathrm{H}), 2.86(\mathrm{q}, J=7.5 \mathrm{~Hz}$, 2H), $7.58(\mathrm{~m}, 2 \mathrm{H}), 7.70(\mathrm{dt}, J=7.0$ and $1.3 \mathrm{~Hz}, 1 \mathrm{H}), 7.97(\mathrm{~d}, J=8.0 \mathrm{~Hz}, 1 \mathrm{H})$ and 9.23 $\operatorname{ppm}(\mathrm{s}, 1 \mathrm{H}) ;{ }^{13} \mathrm{C}$ NMR $\delta 14.5,29.0,33.2,123.1,126.5,127.0,128.2,131.0,131.5,132.2$, 150.7, 152.8 and $205.9 \mathrm{ppm}$; HRMS $\left(\mathrm{EI}^{+}\right) 199.0995$ (cal for $\mathrm{C}_{13} \mathrm{H}_{13} \mathrm{NO}$ 199.0997); IR $1197,1496,1574,1621,1699\left(v_{\mathrm{CO}}\right)$ and $2971 \mathrm{~cm}^{-1}$.

Methyl 3-pentylisoquinoline-4-carboxylate (3i): Viscous liquid; $\mathrm{R}_{\mathrm{f}}=0.45(15 \%$ ethyl acetate in hexanes); ${ }^{1} \mathrm{H}$ NMR $\delta 0.87(\mathrm{t}, J=7.25 \mathrm{~Hz}, 3 \mathrm{H}), 1.34(\mathrm{~m}, 4 \mathrm{H}), 1.80(\mathrm{~m}, 2 \mathrm{H})$, $2.90(\mathrm{~m}, 2 \mathrm{H}), 4.03(\mathrm{~s}, 1 \mathrm{H}), 7.55(\mathrm{dt}, J=7.5$ and $1.3 \mathrm{~Hz}, 1 \mathrm{H}), 7.69(\mathrm{dt}, J=8.25$ and 1.3 $\mathrm{Hz}, 1 \mathrm{H}), 7.78(\mathrm{~d}, J=8.0 \mathrm{~Hz}, 1 \mathrm{H}), 7.93(\mathrm{~d}, J=7.5 \mathrm{~Hz}, 1 \mathrm{H})$ and $9.22 \mathrm{ppm}(\mathrm{s}, 1 \mathrm{H}) ;{ }^{13} \mathrm{C} \mathrm{NMR}$ $\delta 14.0,22.5,29.9,31.8,36.2,52.5,123.8,126.4,127.1,128.1,131.8,133.4,153.1,153.4$ and 168.9 ppm; HRMS (EI $\left.{ }^{+}\right) 257.1411$ (cal for $\mathrm{C}_{16} \mathrm{H}_{19} \mathrm{NO}_{2}$ 257.1416); IR 1223, 1435, $1575,1622,1729$ and $2954 \mathrm{~cm}^{-1}$.

4-Butyl-3-(hex-1-ynyl)isoquinoline (3j): Viscous liquid; $\mathrm{R}_{\mathrm{f}}=0.39$ (15\% ethyl acetate in hexanes); ${ }^{1} \mathrm{H}$ NMR $\delta$ 0.93-0.99 (m, 6H), 1.48-1.53 (m, 4H), 1.63-1.66 (m, 4H), $2.53(\mathrm{t}, J=7.3 \mathrm{~Hz}, 2 \mathrm{H}), 3.20(\mathrm{t}, J=8.0 \mathrm{~Hz}, 2 \mathrm{H}), 7.52(\mathrm{t}, J=7.8 \mathrm{~Hz}, 1 \mathrm{H}), 7.70(\mathrm{t}, J=7.5$ $\mathrm{Hz}, 1 \mathrm{H}), 7.88(\mathrm{~d}, J=7.5 \mathrm{~Hz}, 1 \mathrm{H}), 7.94(\mathrm{~d}, J=8.5 \mathrm{~Hz}, 1 \mathrm{H})$ and $9.00(\mathrm{~s}, 1 \mathrm{H}) ;{ }^{13} \mathrm{C}$ NMR $\delta$ 13.6, 14.0, 19.3, 22.1, 23.1, 29.2, 30.6, 32.4, 79.5, 94.4, 123.3, 127.0, 127.4, 128.5, 130.7, 134.8, 135.5 and 150.3 ppm; HRMS (EI $\left.{ }^{+}\right) 265.1832$ (cal for $\mathrm{C}_{19} \mathrm{H}_{23} \mathrm{~N}$ 265.183); IR 1454, 1505,1572 , and $2957 \mathrm{~cm}^{-1}$.

4-Ethyl-3-(oct-5-ynyl)isoquinoline (3ka): Viscous liquid; $\mathrm{R}_{\mathrm{f}}=0.41$ (15\% ethyl acetate in hexanes); ${ }^{1} \mathrm{H}$ NMR $\delta 1.08(\mathrm{t}, J=7.5 \mathrm{~Hz}, 3 \mathrm{H}), 1.29(\mathrm{t}, J=7.5 \mathrm{~Hz}, 3 \mathrm{H})$, 1.61-1.66 (m, 2 H), 1.84-1.89 (m, 2 H), 2.10-2.15 (m, 2H), 2.20-2.22 (m, 2H), 2.99 (t, $J=$ 
$8.0 \mathrm{~Hz}, 2 \mathrm{H}), 3.08$ (q, $J=15.3$ and $7.3 \mathrm{~Hz}, 2 \mathrm{H}), 7.53(\mathrm{t}, J=7.5 \mathrm{~Hz}, 1 \mathrm{H}), 7.70(\mathrm{t}, J=7.5$ $\mathrm{Hz}, 1 \mathrm{H}), 7.93(\mathrm{~d}, J=8.0 \mathrm{~Hz}, 1 \mathrm{H}), 7.99(\mathrm{~d}, J=8.5 \mathrm{~Hz}, 1 \mathrm{H})$ and $9.08(\mathrm{~s}, 1 \mathrm{H}) ;{ }^{13} \mathrm{C} \mathrm{NMR} \delta$ $12.4,14.3,15.0,18.7,20.9,29.2,29.6,34.4,79.2,81.9,122.9,126.0,127.2,128.4,130.0$ 130.5, 135.3, 150.0 and 151.7; HRMS (EI $\left.{ }^{+}\right) 265.1830$ (cal for $\mathrm{C}_{19} \mathrm{H}_{23} \mathrm{~N}$ 265.1830); IR $1242,1454,1576,1621$, and $2935 \mathrm{~cm}^{-1}$.

3-Ethyl-4-(oct-5-ynyl)isoquinoline (3kb): Viscous liquid; $\mathrm{R}_{\mathrm{f}}=0.36$ (15\% ethyl acetate in hexanes); ${ }^{1} \mathrm{H}$ NMR $\delta 1.08(\mathrm{t}, J=7.5 \mathrm{~Hz}, 3 \mathrm{H}), 1.35(\mathrm{t}, J=7.5 \mathrm{~Hz}, 3 \mathrm{H})$, 1.68-1.69 (m, 2H), 1.74-1.77 (m, 2H), 2.13-2.15 (m, 2H), 2.24-2.26 (m, 2H), 3.00-3.05 (m, 4H), $7.52(\mathrm{t}, J=7.5 \mathrm{~Hz}, 1 \mathrm{H}), 7.68(\mathrm{t}, J=7.5 \mathrm{~Hz}, 1 \mathrm{H}), 7.92(\mathrm{~d}, J=8.4 \mathrm{~Hz}, 1 \mathrm{H}), 7.99$ $(\mathrm{d}, J=8.4 \mathrm{~Hz}, 1 \mathrm{H})$ and $9.08(\mathrm{~s}, 1 \mathrm{H}) ;{ }^{13} \mathrm{C} \mathrm{NMR} \delta_{\mathrm{C}}=12.4,14.3,14.6,18.4,27.2,28.1$, $29.2,29.6,78.8,82.3,123.0,125.9,127.2,128.0,128.3,130.4,135.5,149.9$ and 153.6.

3-Phenylisoquinoline (3l): Pale green solid; $\mathrm{mp}=93{ }^{\circ} \mathrm{C} ; \mathrm{R}_{\mathrm{f}}=0.58$ (15\% ethyl acetate in hexanes); ${ }^{1} \mathrm{H}$ NMR $\delta 7.41(\mathrm{t}, J=7.5 \mathrm{~Hz}, 1 \mathrm{H}), 7.50(\mathrm{t}, J=7.3 \mathrm{~Hz}, 2 \mathrm{H}), 7.56(\mathrm{dt}$, $J=7.0$ and $1.0 \mathrm{~Hz}, 1 \mathrm{H}), 7.67(\mathrm{~m}, 1 \mathrm{H}), 7.85(\mathrm{~d}, J=8.5 \mathrm{~Hz}, 1 \mathrm{H}), 7.97(\mathrm{~d}, J=8.5 \mathrm{~Hz}, 1 \mathrm{H})$, $8.05(\mathrm{~s}, 1 \mathrm{H}), 8.12(\mathrm{dd}, J=8.3$ and $1.3 \mathrm{~Hz}, 2 \mathrm{H})$ and $9.33 \mathrm{ppm}(\mathrm{s}, 1 \mathrm{H}) ;{ }^{13} \mathrm{C} \mathrm{NMR} \delta 116.3$, $126.6,126.7,126.8,127.3,127.4,128.3,128.5,130.3,136.4,139.2,150.9$ and 152.1 ppm; HRMS (EI $\left.{ }^{+}\right) 205.0892$ (cal for $\mathrm{C}_{15} \mathrm{H}_{11} \mathrm{~N}$ 205.0891); IR 1453, 1579, 1622 and 3058 $\mathrm{cm}^{-1}$.

3-Butylisoquinoline (3m): Viscous liquid; $\mathrm{R}_{\mathrm{f}}=0.45$ (15\% ethyl acetate in hexanes); ${ }^{1} \mathrm{H}$ NMR $\delta 0.94$ (t, $\left.J=7.5 \mathrm{~Hz}, 3 \mathrm{H}\right), 1.40(\mathrm{~m}, 2 \mathrm{H}), 1.78(\mathrm{~m}, 2 \mathrm{H}), 2.94(\mathrm{t}, J=7.5$ Hz, 2H), $7.48(\mathrm{~s}, 1 \mathrm{H}), 7.53(\mathrm{t}, J=7.8 \mathrm{~Hz}, 1 \mathrm{H}), 7.65(\mathrm{t}, J=7.3 \mathrm{~Hz}, 1 \mathrm{H}), 7.74(\mathrm{~d}, J=8.0$ $\mathrm{Hz}, 1 \mathrm{H}), 7.93(\mathrm{~d}, J=8.0 \mathrm{~Hz}, 1 \mathrm{H})$ and $9.20(\mathrm{~s}, 1 \mathrm{H}) ;{ }^{13} \mathrm{C} \mathrm{NMR} \delta 14.0,22.5,32.1,37.4$, 
$118.3,126.1,126.5,127.0,127.6,130.6,136.7,151.6$, and 155.2; HRMS (EI $\left.{ }^{+}\right) 185.1206$ (cal for $\mathrm{C}_{13} \mathrm{H}_{15} \mathrm{~N}$ 185.1204).

4-(Isoquinolin-3-yl)butanenitrile (3n): White solid; $\mathrm{mp}=98{ }^{\circ} \mathrm{C} ; \mathrm{R}_{\mathrm{f}}=0.08(15 \%$ ethyl acetate in hexanes); ${ }^{1} \mathrm{H}$ NMR $\delta$ 2.18-2.44 (m, 2H), 2.39 (t, $\left.J=6.8 \mathrm{~Hz}, 2 \mathrm{H}\right), 3.10$ (t, $J=7.5 \mathrm{~Hz}, 2 \mathrm{H}), 7.56(\mathrm{~s}, 1 \mathrm{H}), 7.59(\mathrm{t}, J=8.0 \mathrm{~Hz}, 1 \mathrm{H}), 7.71(\mathrm{t}, J=7.0 \mathrm{~Hz}, 1 \mathrm{H}), 7.78(\mathrm{~d}, J$ $=8.5 \mathrm{~Hz}, 1 \mathrm{H}), 7.96(\mathrm{~d}, J=8.0 \mathrm{~Hz}, 1 \mathrm{H})$ and $9.22(\mathrm{~s}, 1 \mathrm{H}) ;{ }^{13} \mathrm{C}$ NMR $\delta 16.5,25.2,35.7$, 119.3, 121.1, 126.6, 126.8, 128.5, 133.2, 137.6 and 149.9 ppm; ; HRMS $\left(\mathrm{EI}^{+}\right) 196.0997$ (cal for $\mathrm{C}_{13} \mathrm{H}_{12} \mathrm{~N}_{2}$ 196.1000); IR 1465, 2248 and $2941 \mathrm{~cm}^{-1}$.

1,3-Dihydro-7,8-diphenylfuro[3,4-g]isoquinoline (3o): White solid; $\mathrm{mp}=225$ ${ }^{\circ} \mathrm{C} ; \mathrm{R}_{\mathrm{f}}=0.18$ ( $15 \%$ ethyl acetate in hexanes ); ${ }^{1} \mathrm{H}$ NMR $\delta 6.05(\mathrm{~s}, 2 \mathrm{H}), 6.90(\mathrm{~s}, 1 \mathrm{H}), 7.17$ (m, 5H), $7.25(\mathrm{~d}, J=4.5 \mathrm{~Hz}, 1 \mathrm{H}), 7.32(\mathrm{~m}, 5 \mathrm{H})$ and $9.09 \mathrm{ppm}(\mathrm{s}, 1 \mathrm{H}) ;{ }^{13} \mathrm{C} \mathrm{NMR} \delta$ 101.7, 8102.0, 103.0, 124.8, 127.0, 127.3, 127.6, 128.4, 130.1, 130.6, 131.1, 134.5, 137.5, 140.6, 148.1, 149.3, 149.8 and $151.3 \mathrm{ppm}$; IR 1207, 1454 and $2914 \mathrm{~cm}^{-1}$.

Phenyl(3-phenylisoquinolin-4-yl)methanone (3p): White solid; $\mathrm{mp}=116{ }^{\circ} \mathrm{C}$; $\mathrm{R}_{\mathrm{f}}=0.30$ ( $15 \%$ ethyl acetate in hexanes ); ${ }^{1} \mathrm{H}$ NMR $\delta$ 7.18-7.26 (m, 5H), $7.40(\mathrm{t}, J=7.5$ Hz, 1H), 7.59-7.68 (m, 6H), $7.73(\mathrm{~d}, J=4.5 \mathrm{~Hz}, 1 \mathrm{H}), 8.07(\mathrm{~d}, J=9.5 \mathrm{~Hz}, 1 \mathrm{H})$ and 9.45 (s, 1H); ${ }^{13} \mathrm{C}$ NMR $\delta 124.5,127.0,127.6,128.0,128.3,128.5,128.7,129.6,131.5,133.6$, 134.2, 137.4, 139.5, 149.9, 153.4 and 198.0; $\mathrm{HRMS}\left(\mathrm{EI}^{+}\right) 309.1153$ (cal for $\mathrm{C}_{15} \mathrm{H}_{11} \mathrm{~N}$ 309.1154); IR 1229, 1449, 1578, 1618, 1662 and $3058 \mathrm{~cm}^{-1}$.

2,3,4-triphenylpyridine (3q): Pale yellow solid; $\mathrm{mp}=184{ }^{\circ} \mathrm{C} ; \mathrm{R}_{\mathrm{f}}=0.23(10 \%$ ethyl acetate in hexanes ); ${ }^{1} \mathrm{H}$ NMR $\delta 6.86(\mathrm{~d}, J=7.5 \mathrm{~Hz}, 2 \mathrm{H}), 7.01-7.07(\mathrm{~m}, 5 \mathrm{H}), 7.15-$ $7.19(\mathrm{~m}, 6 \mathrm{H}), 7.24-7.26(\mathrm{~m}, 2 \mathrm{H}), 7.32(\mathrm{~d}, J=5.0 \mathrm{~Hz}, 1 \mathrm{H})$ and $8.71(\mathrm{~d}, J=5.0 \mathrm{~Hz}, 1 \mathrm{H})$;

${ }^{13} \mathrm{C}$ NMR $\delta 123.7,126.7,127.4,127.6,127.7,127.9,129.3,129.8,131.3,134.4,137.6$, 
139.2, 140.4, 148.0, 150.0 and 158.2; $\mathrm{HRMS}\left(\mathrm{EI}^{+}\right) 307.1372$ (cal for $\mathrm{C}_{23} \mathrm{H}_{17} \mathrm{~N} 307.1361$ ); IR 1030, 1391, 1430, 1566 and $3053 \mathrm{~cm}^{-1}$.

NOE Experiments. The regiochemistry of most products 3 were established based on the ${ }^{1} \mathrm{H}$ NMR NOE experiments. The results of the NOE experiments are summarized below.
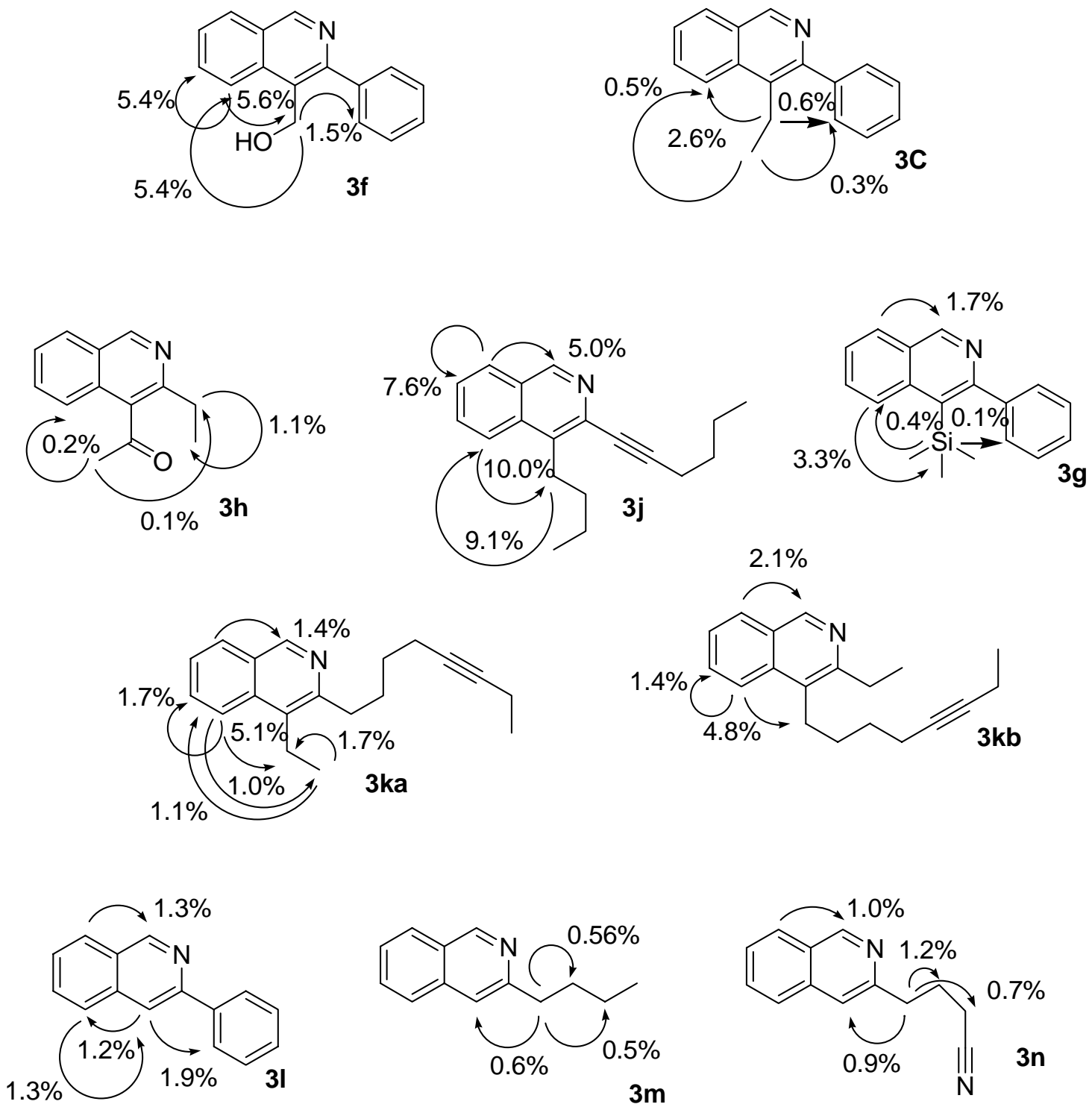
1. Wu, G.; Rhiengold, A. L.; Geib, S. J.; Heck, R. F. Organometallics. 1987, 6, 1941-1946.

2. Maassarani, F.; Pfeffer, M.; Borgne, G. L. J. Chem. Soc.; Chem. Commun. 1987, $565-567$.

3. Roesch, K. R.; Zhang, H.; Larock, R. C. J. Org.Chem. 2001, 66, 8042-8051.

4. Ramakrishna, T. V. V.; Sharp, P. R. Org. Lett. 2003, 5, 877-879.

5. Dai, G.; Larock, R. C. Org. Lett. 2002, 4, 193-196.

6. Kobayashi, T.; Kawate, H.; Kakiuchi, H.; Kato, H. Bull. Chem. Soc. Jpn. 1990, 63, 1937-1942. 


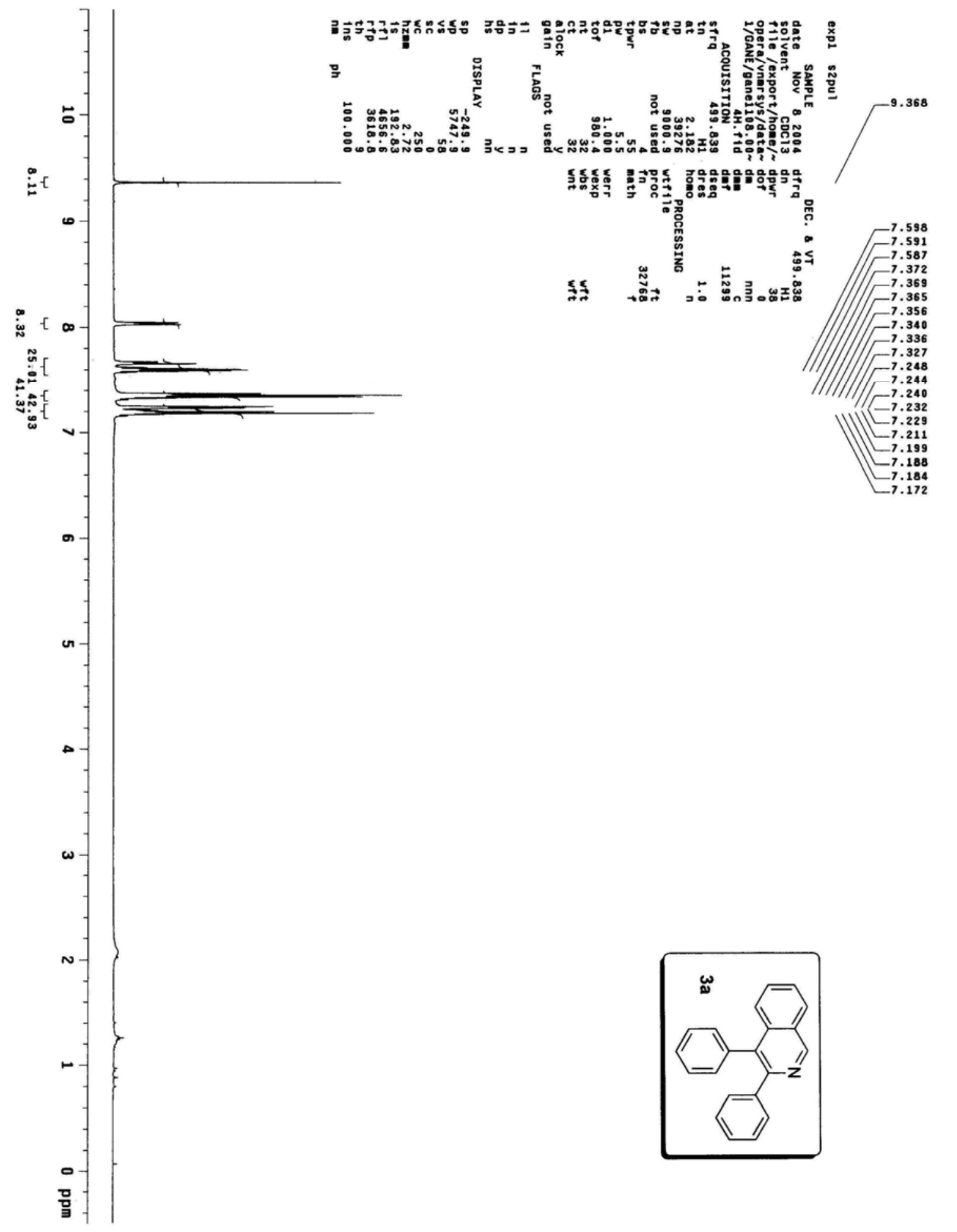




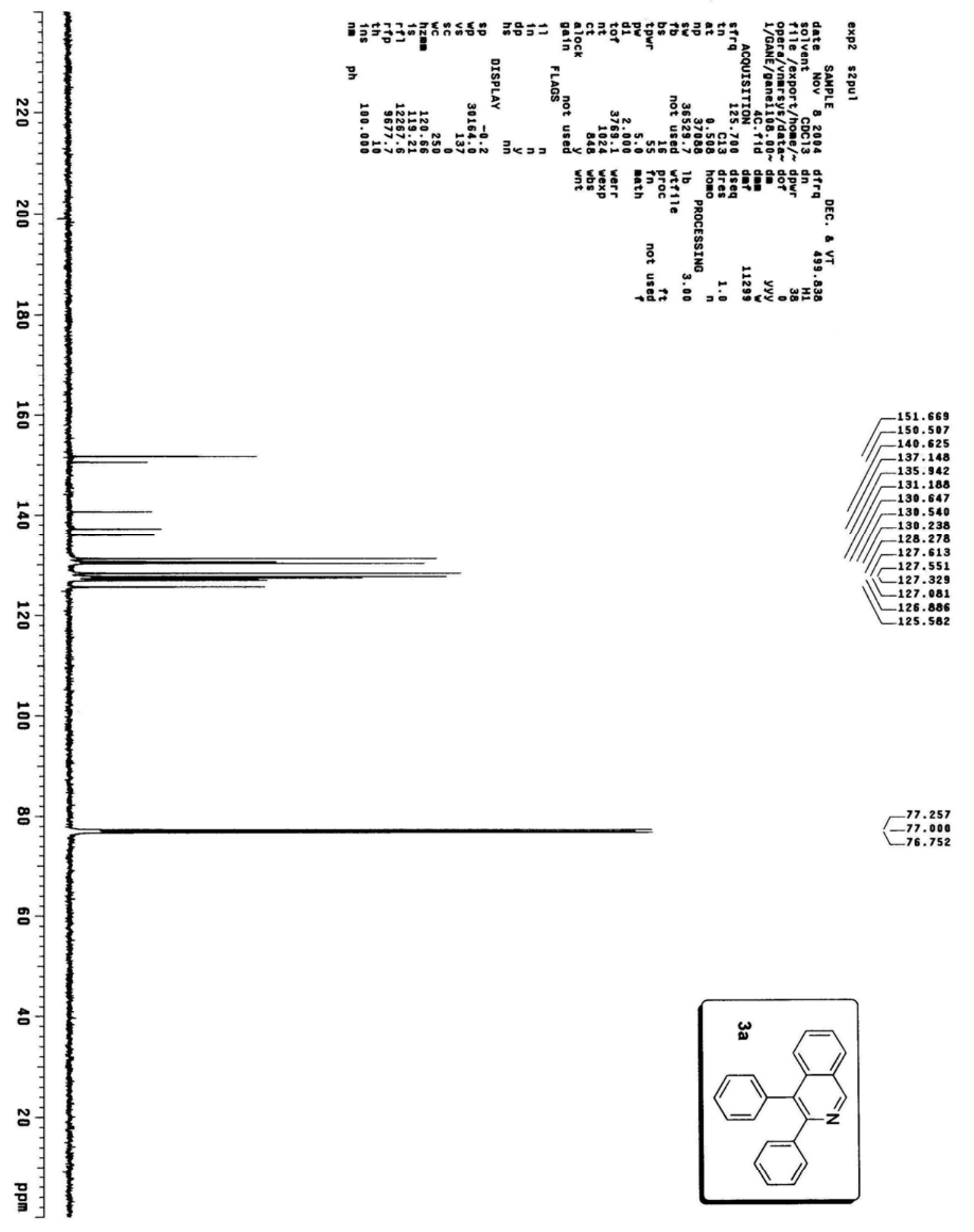



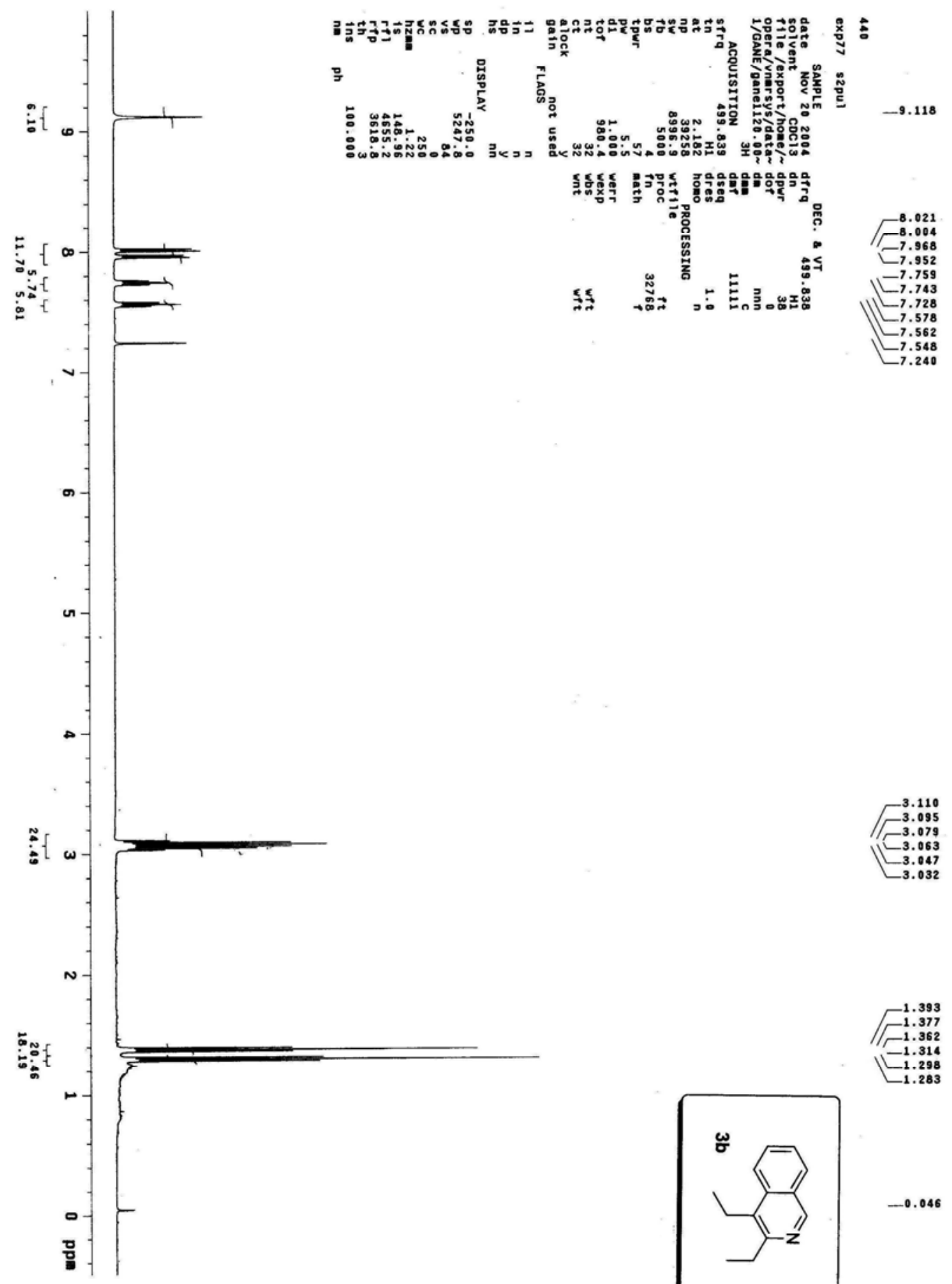

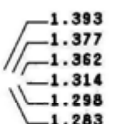

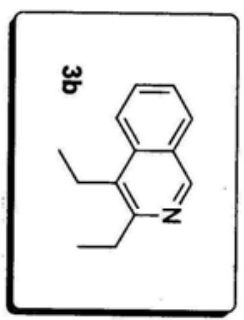

$-0.046$ 


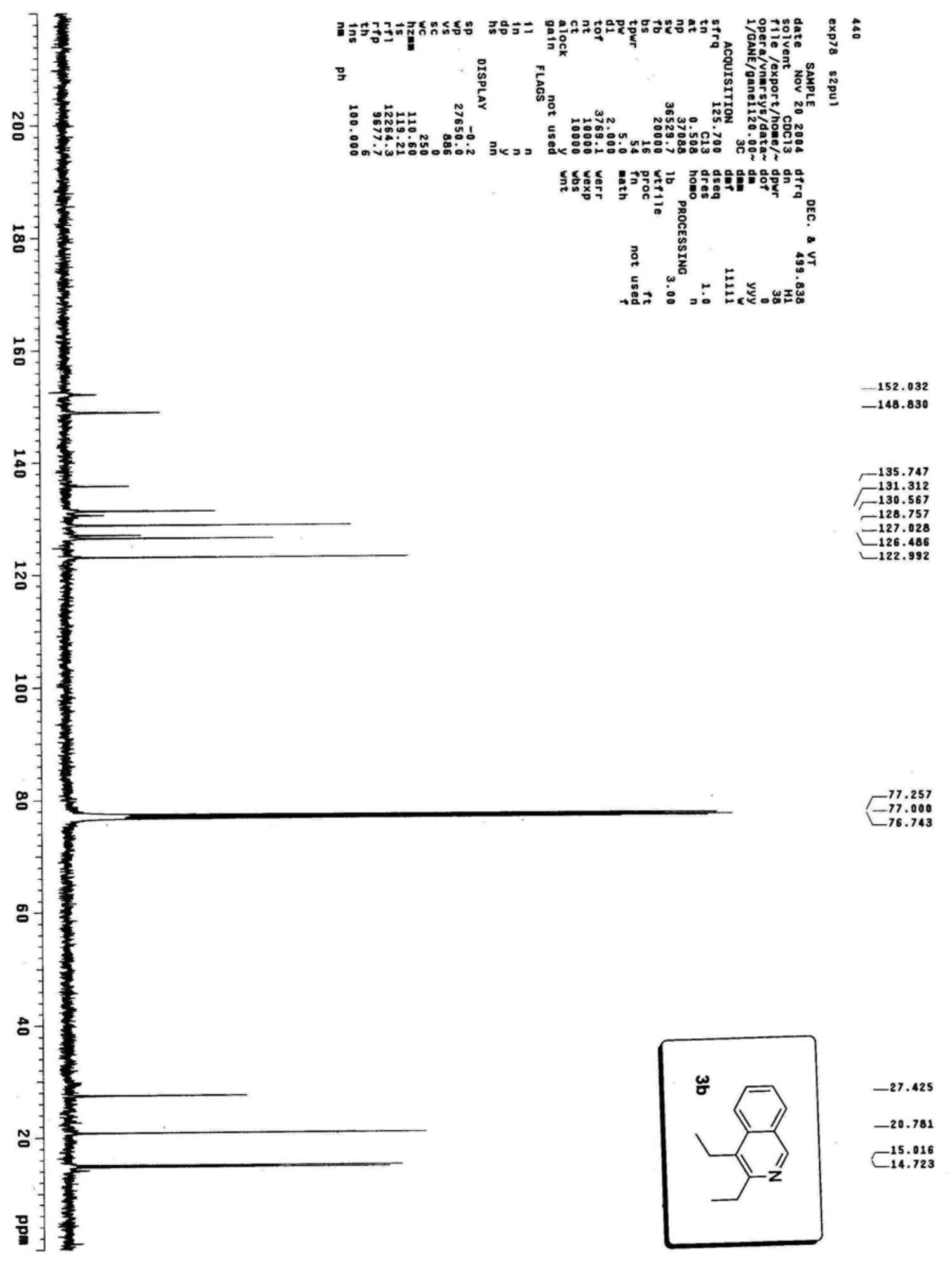




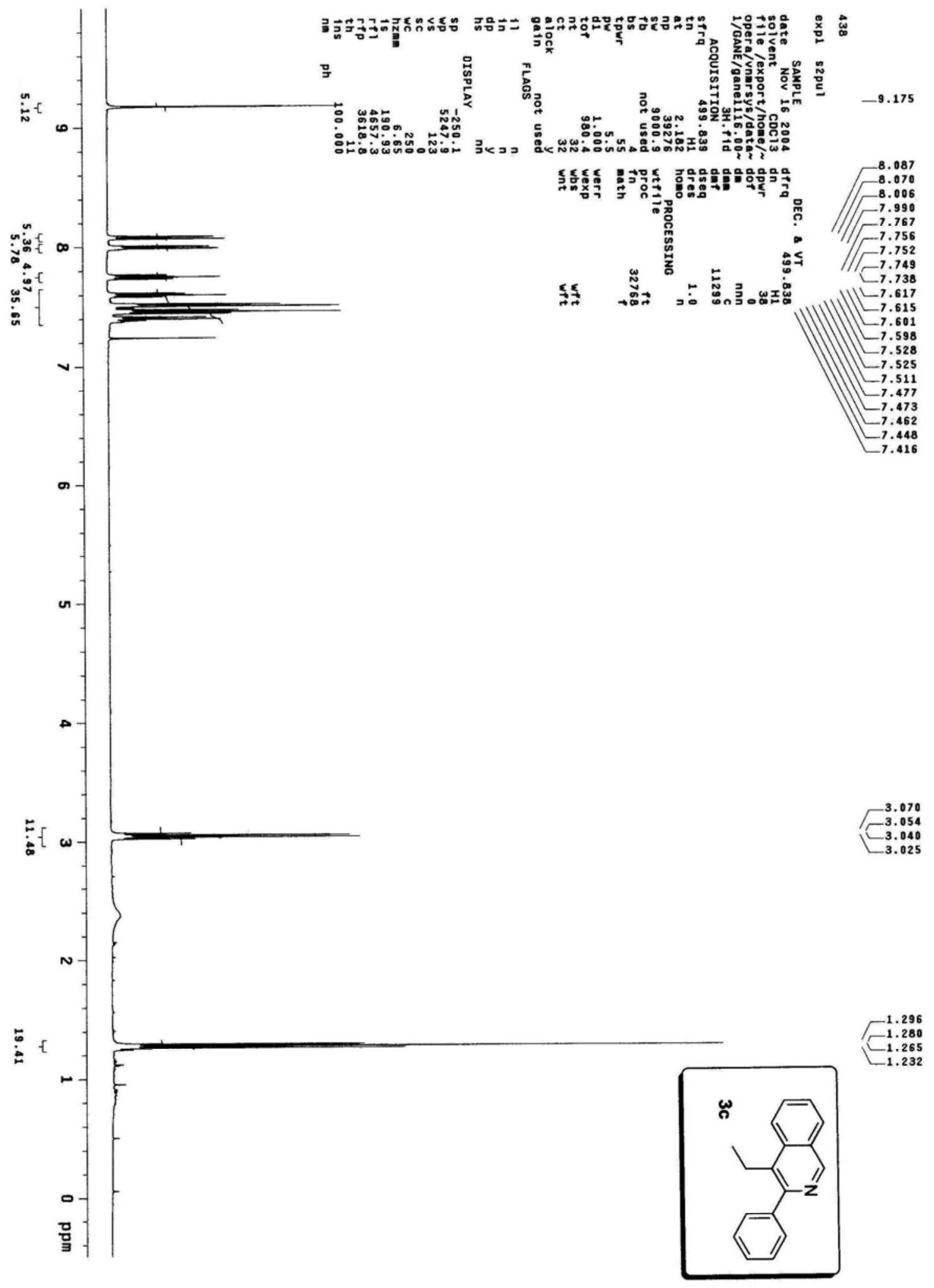



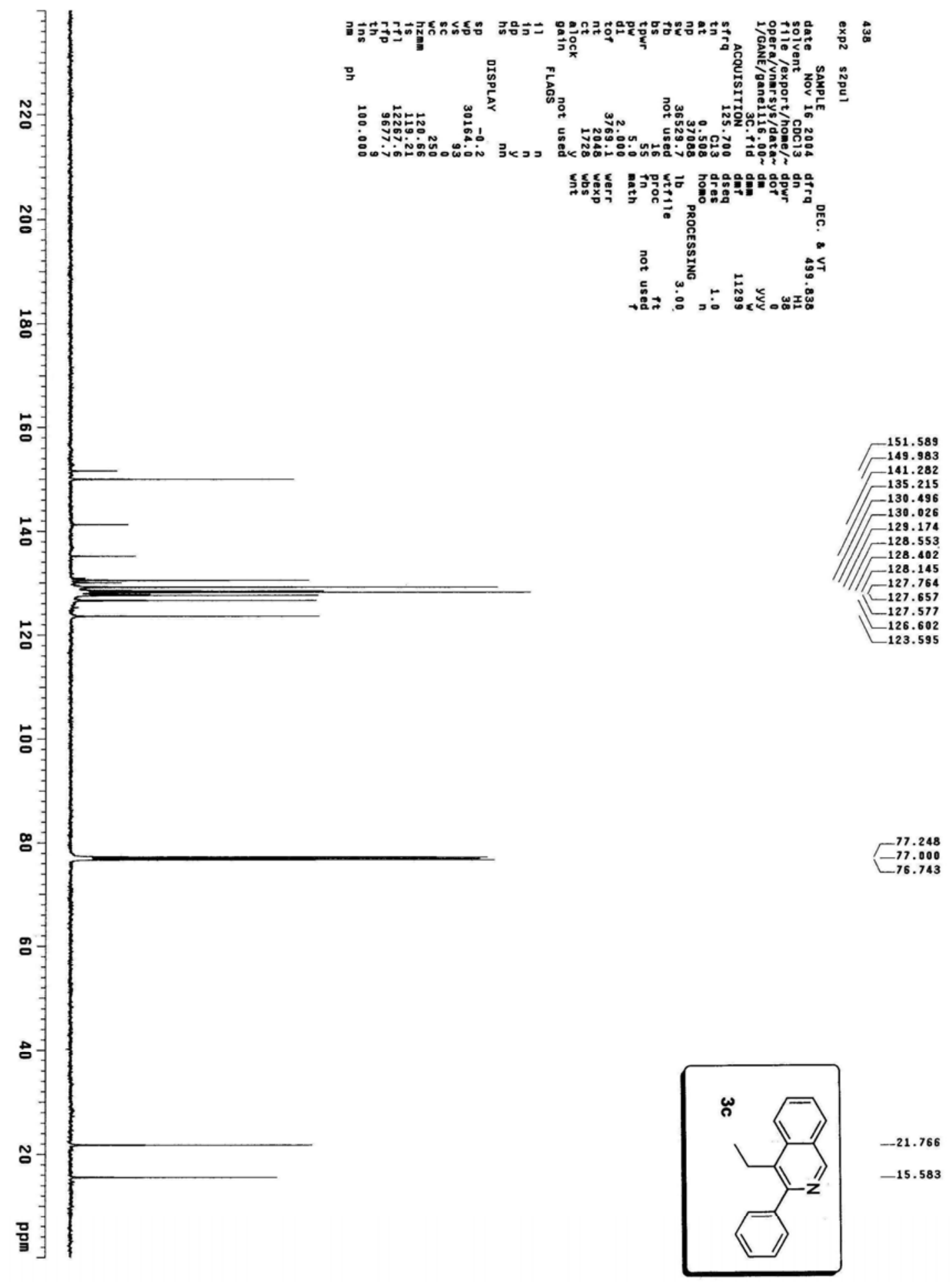

$-21.766$

$-15.583$ 


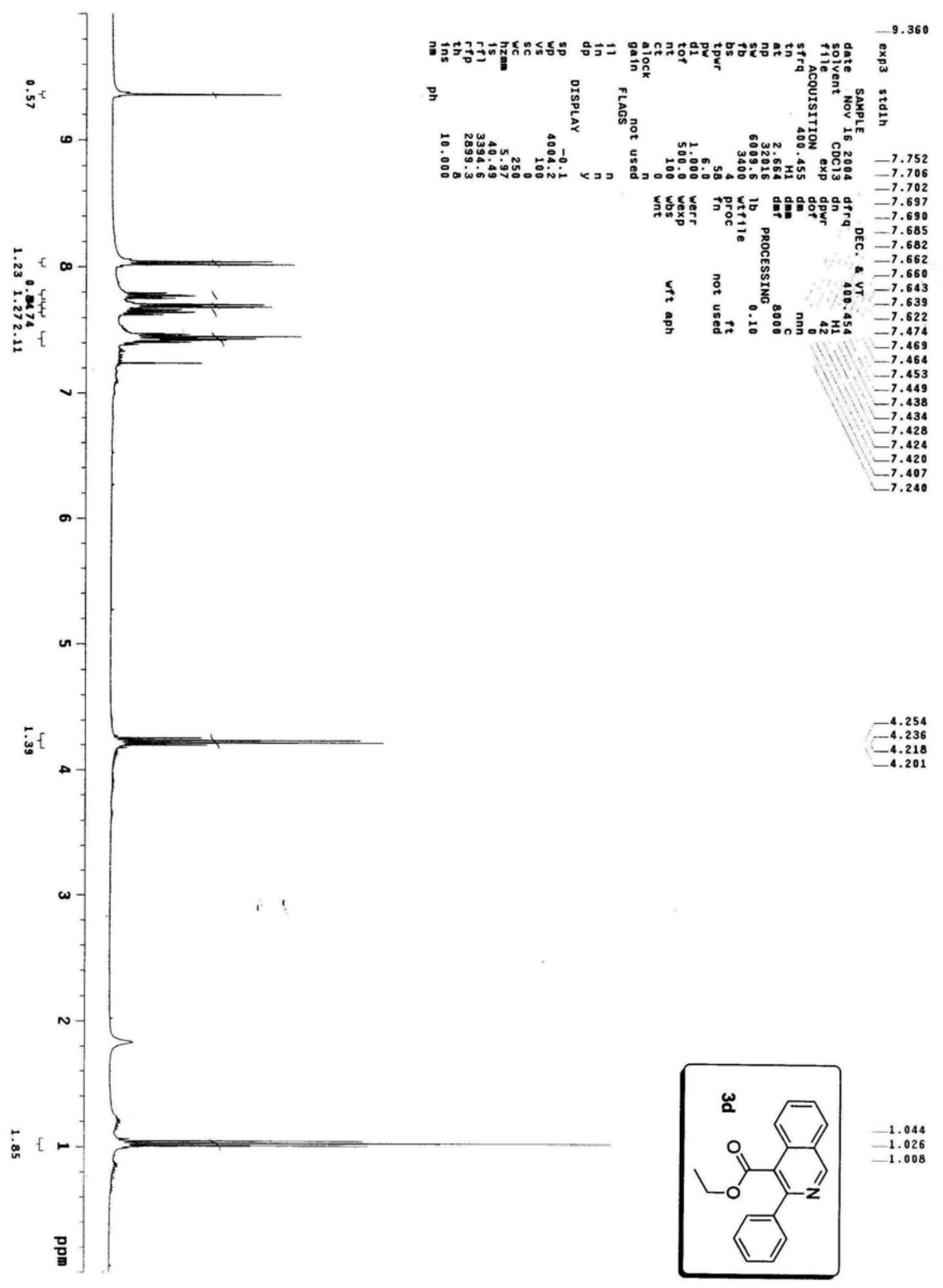



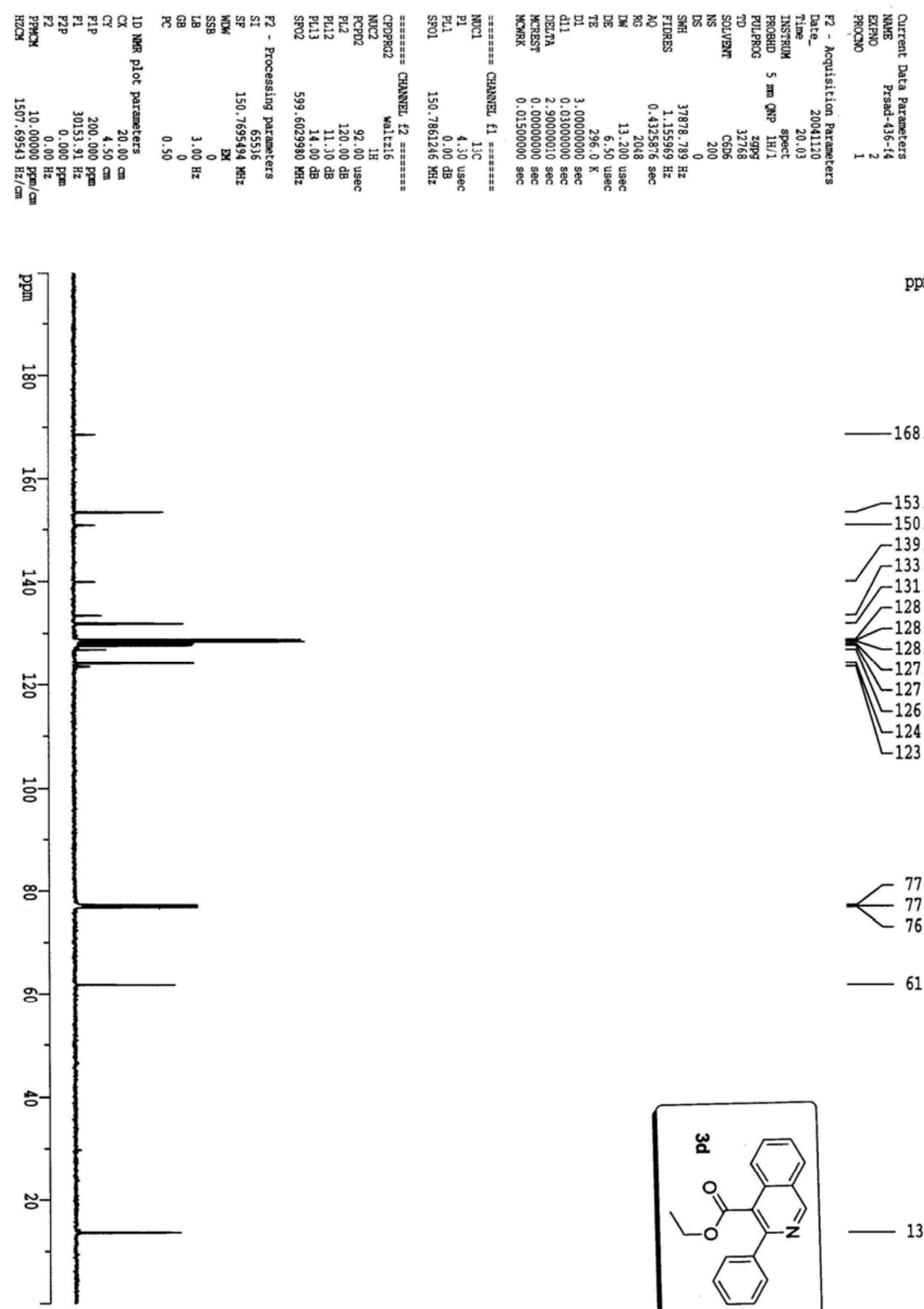

ppm

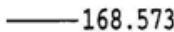

$-153.362$

150.868

139.979

133.420

$-131.812$

$-128.762$

128.564

$-128.382$

$7-127.977$

$-127.638$

L-126.789

-124.127

$-123.425$

77.212

77.000

76.788

61.750

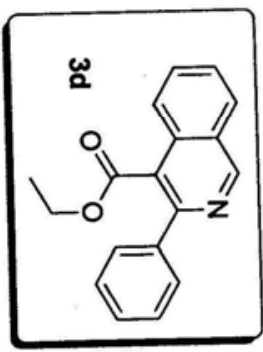

13.632 


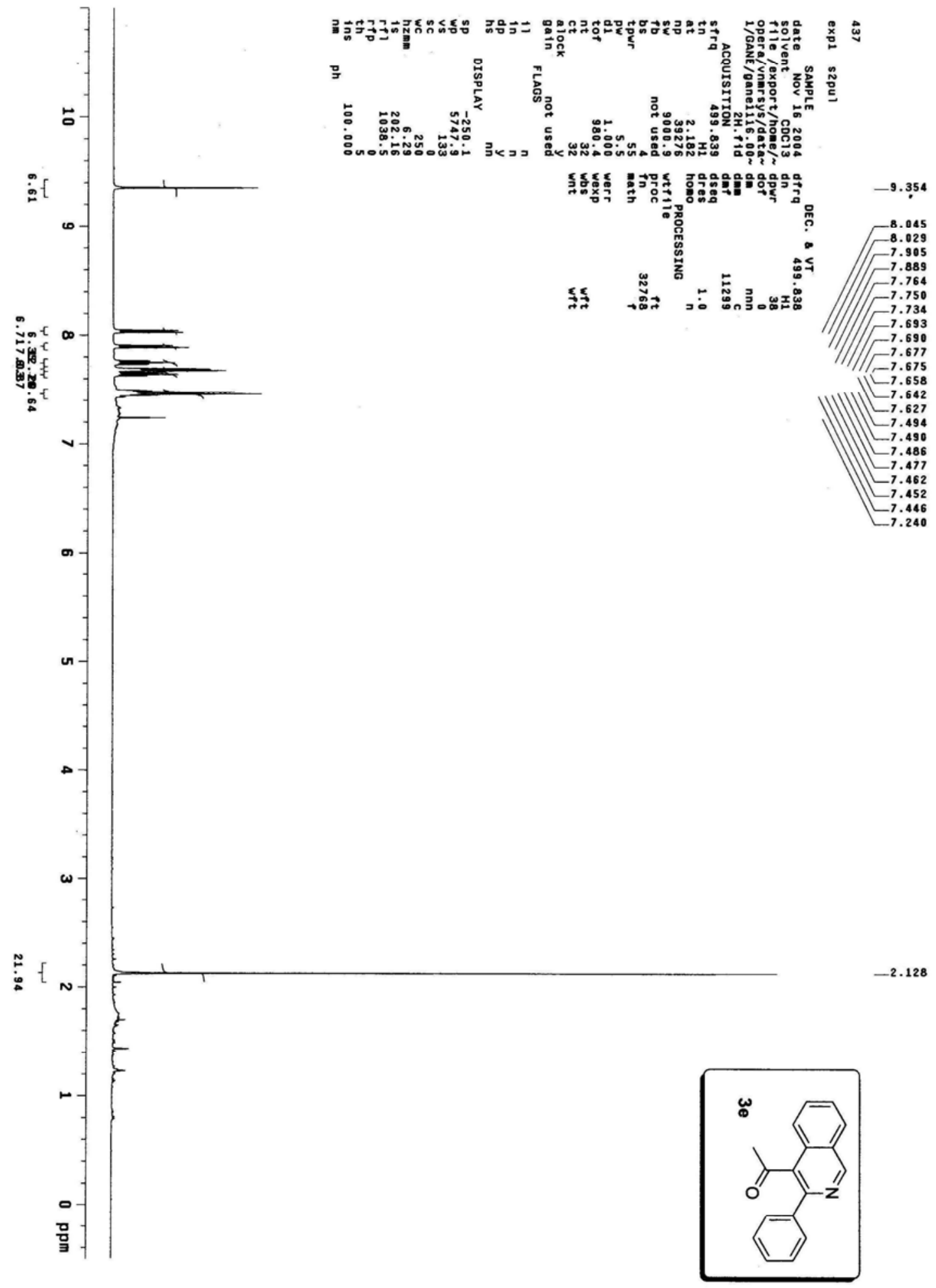




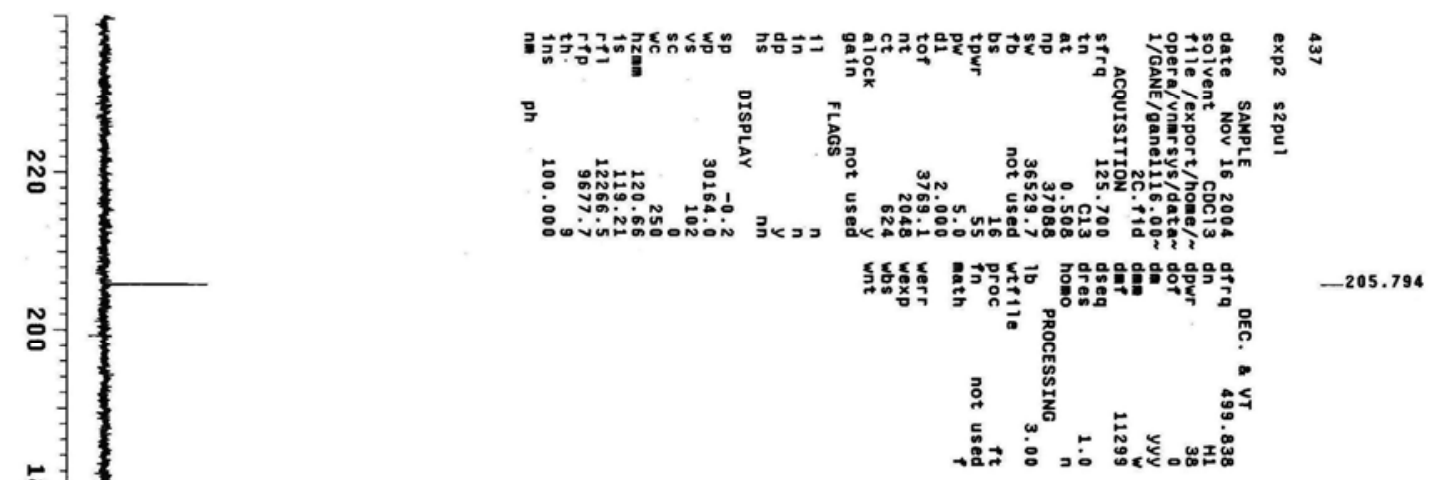

战

음

吕

억

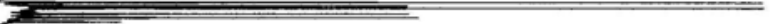

음

$\infty$

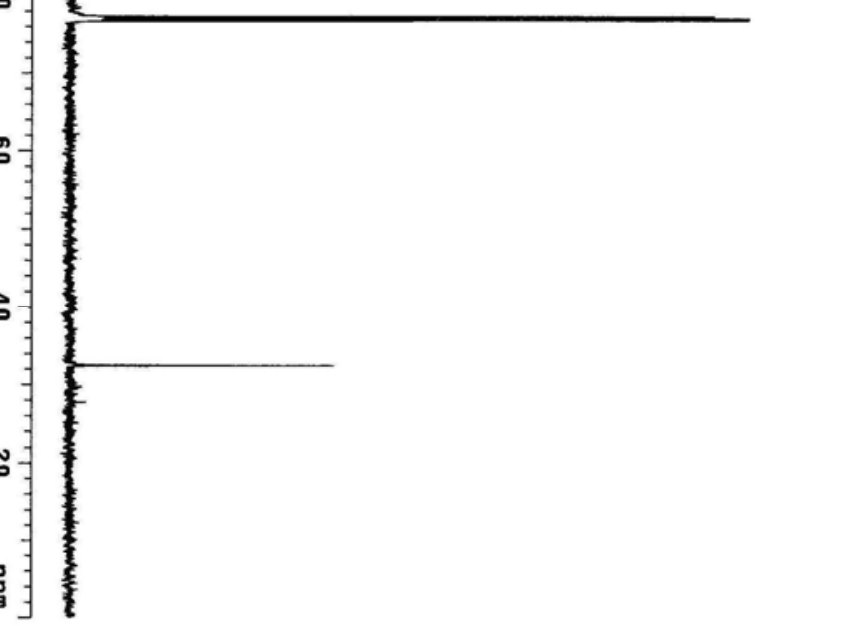

$-153.283$

$-149.194$

${ }_{-139.641}^{132.385}$

$F^{131.755}$

-129.538
-129.068

-129.068
-128.793

$-128.065$

$-127.586$

-127.063
-123.861

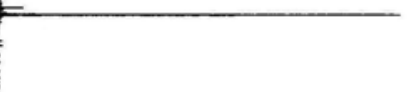

123.861

$\begin{array}{r}77.248 \\ -77.000 \\ \hline\end{array}$

77.000
-76.743

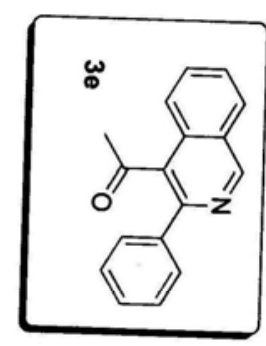

$-32.419$ 

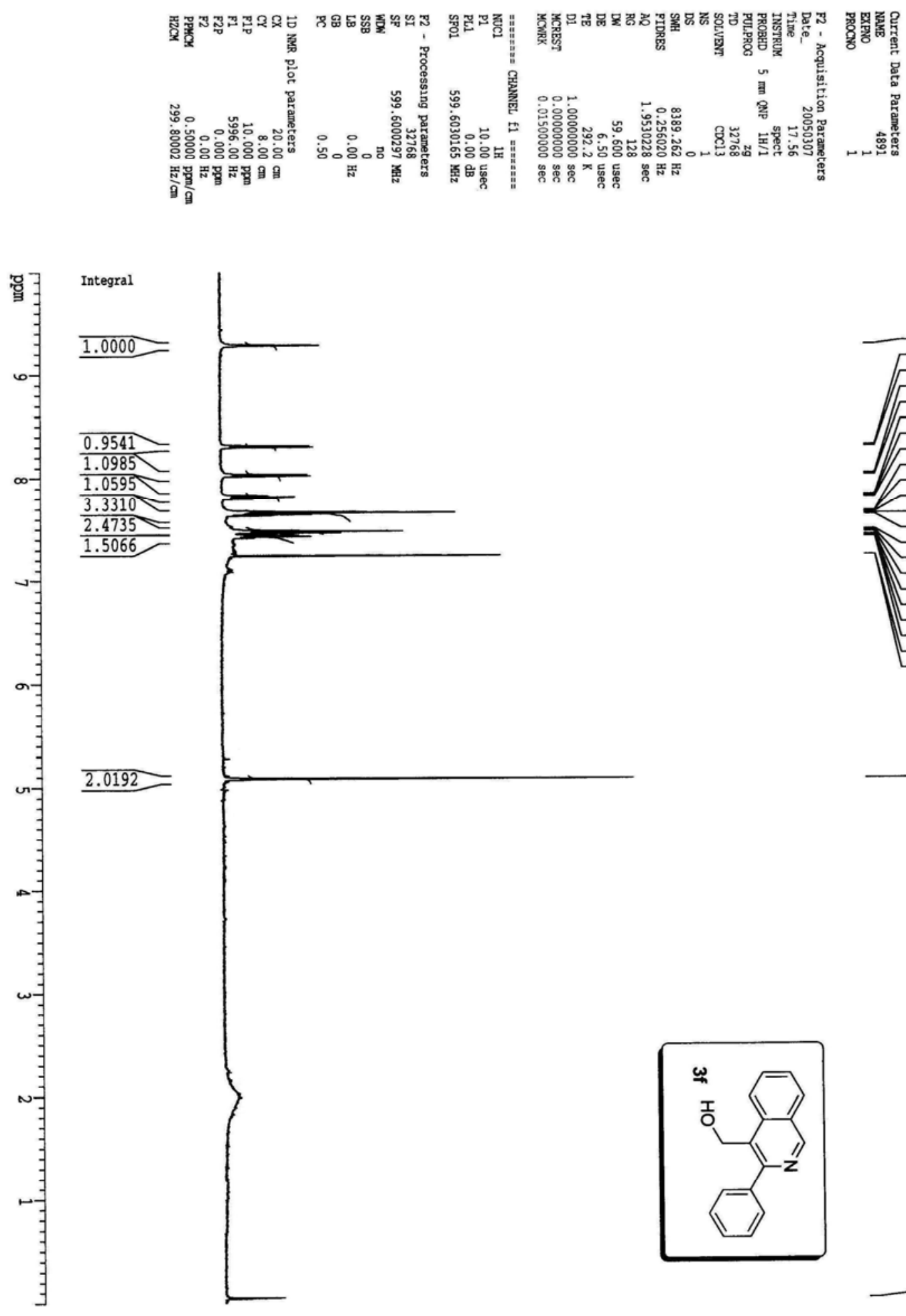

ppm
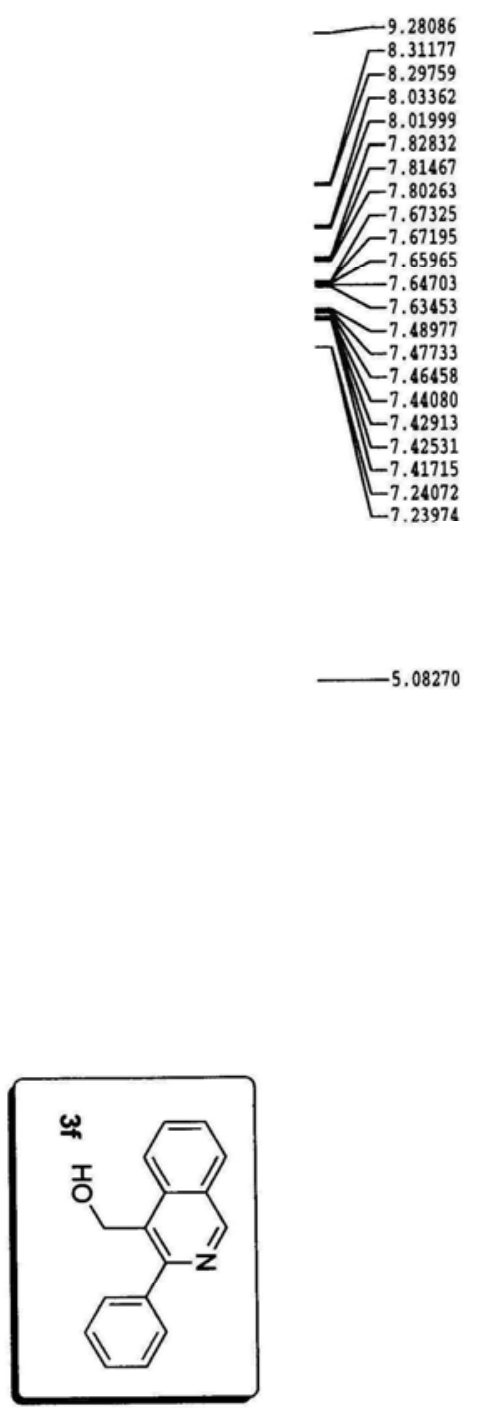

$-0.04864$ 

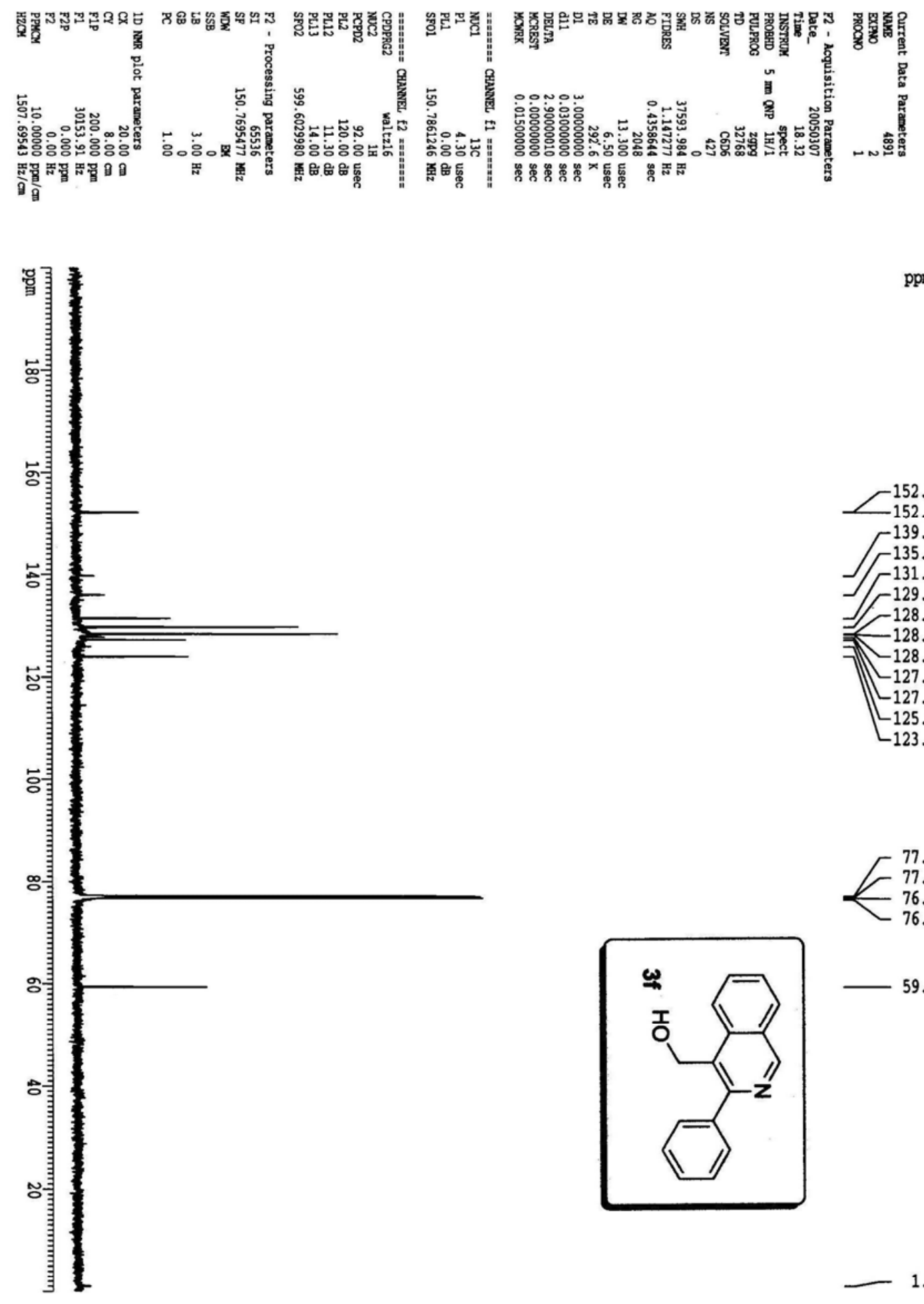

ppm
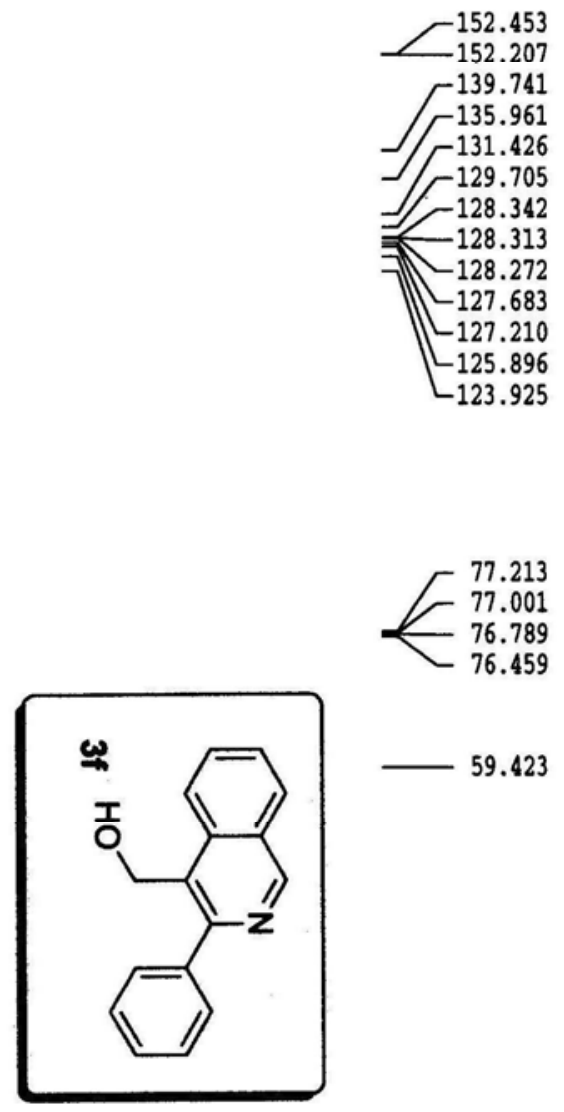

59.423

1.011 


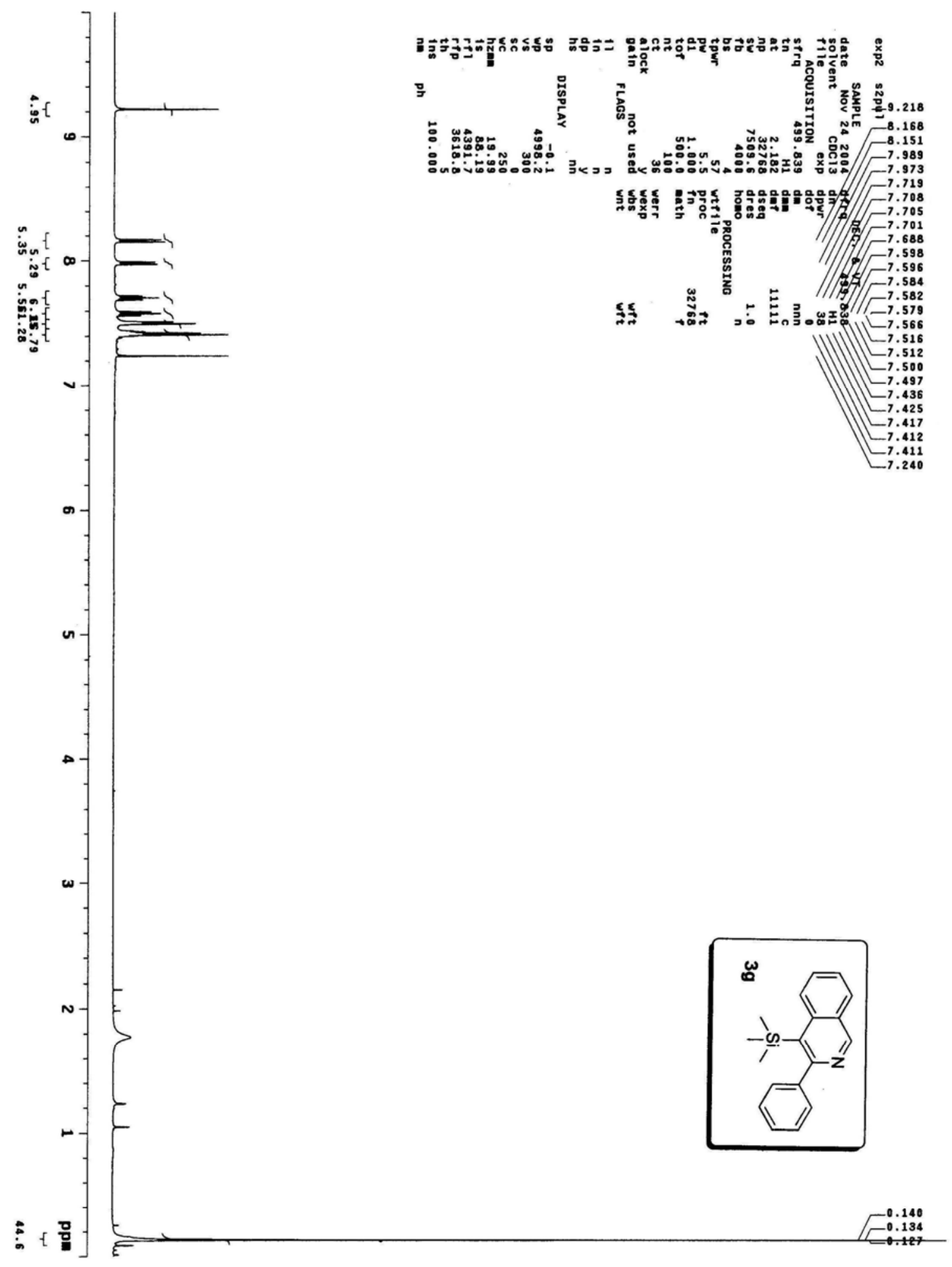



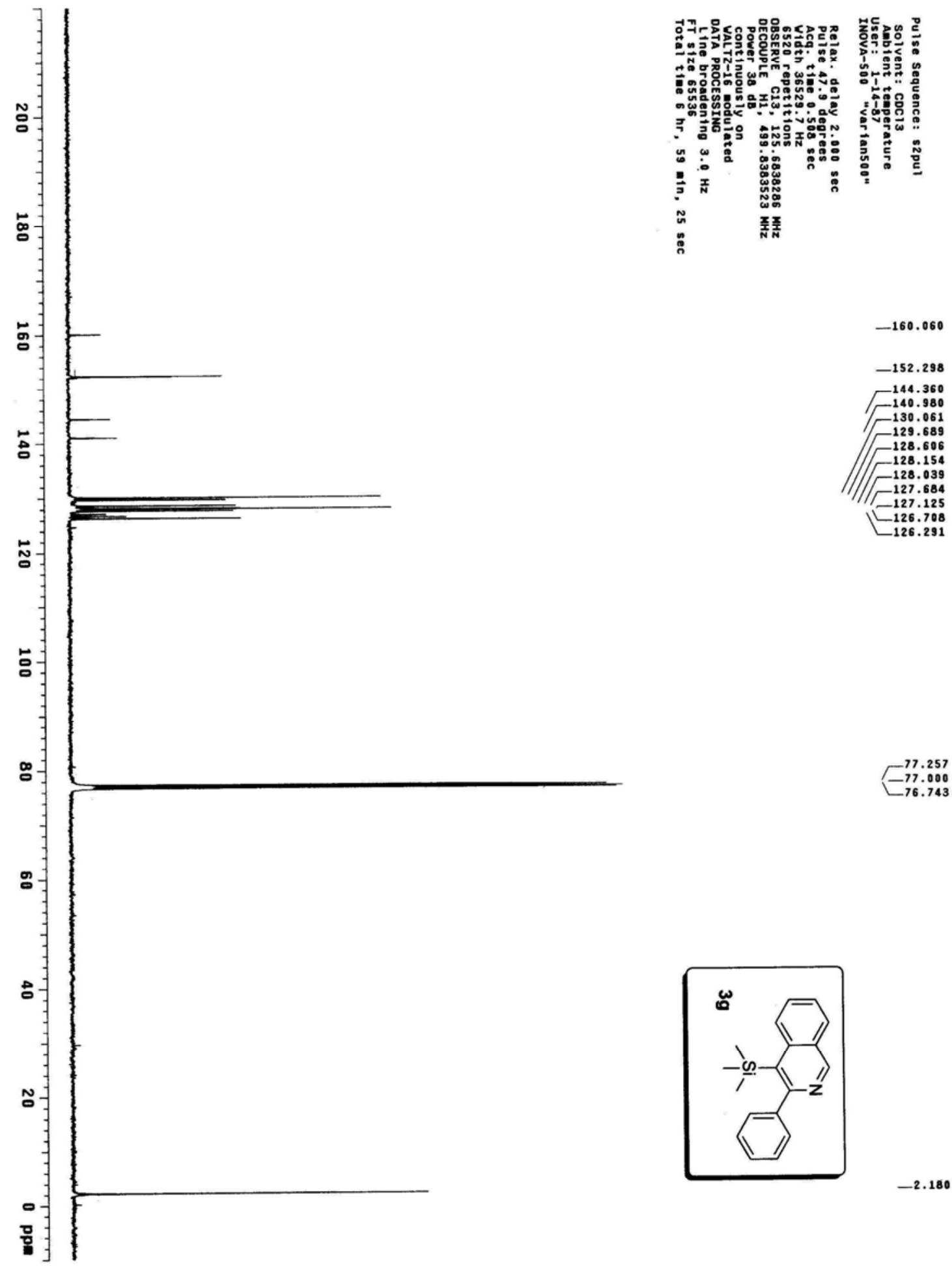

$-2.180$ 


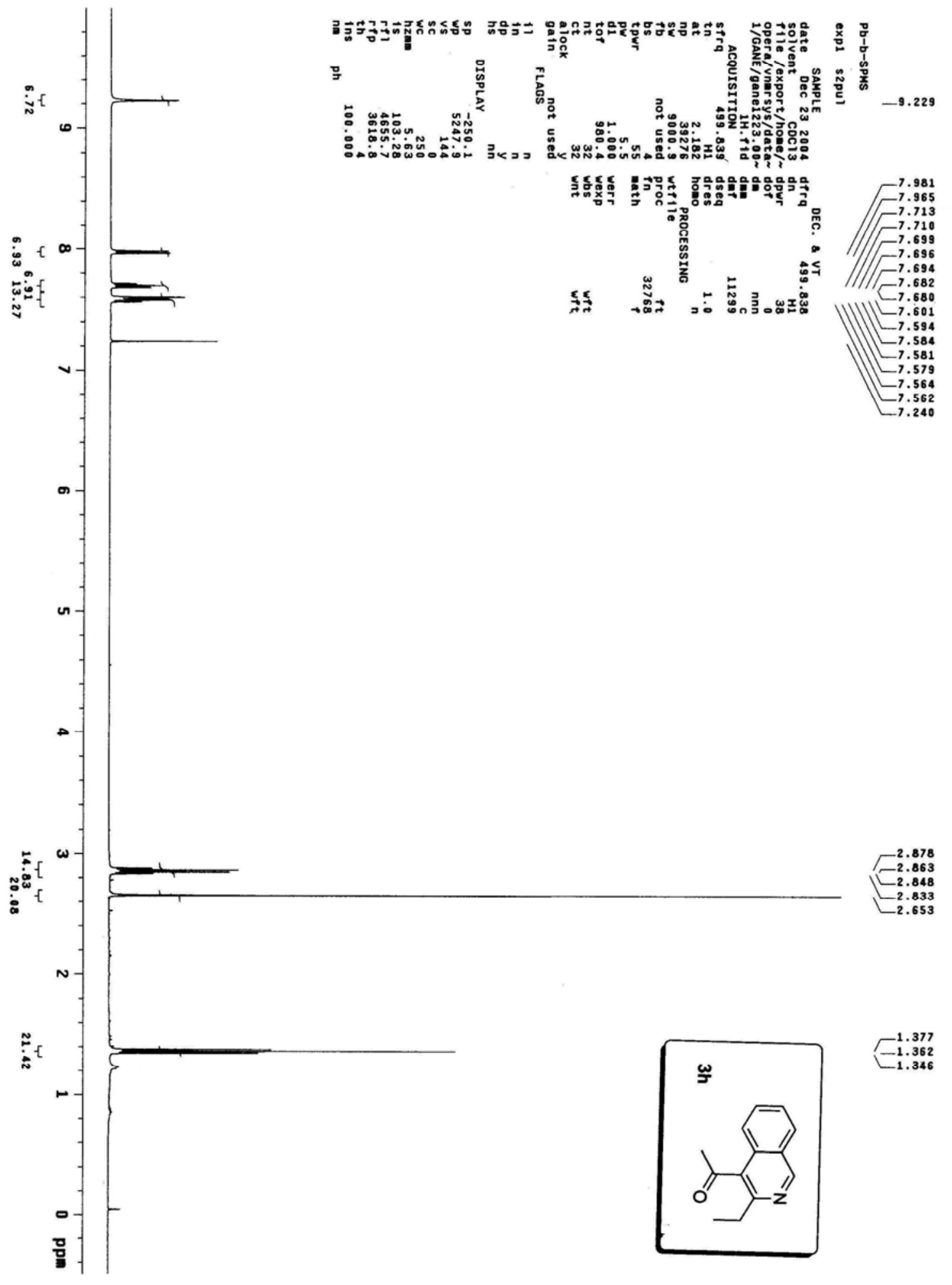




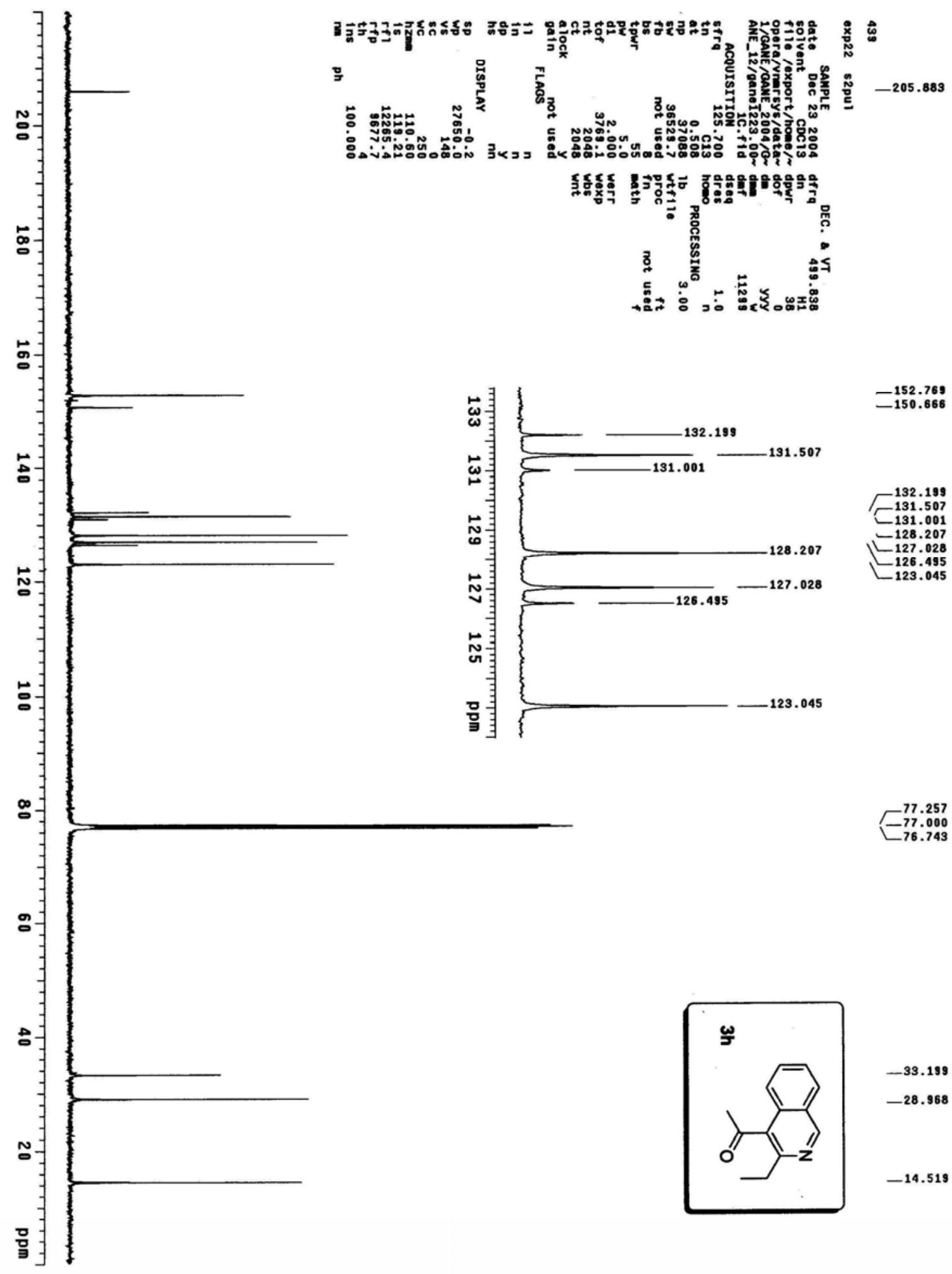




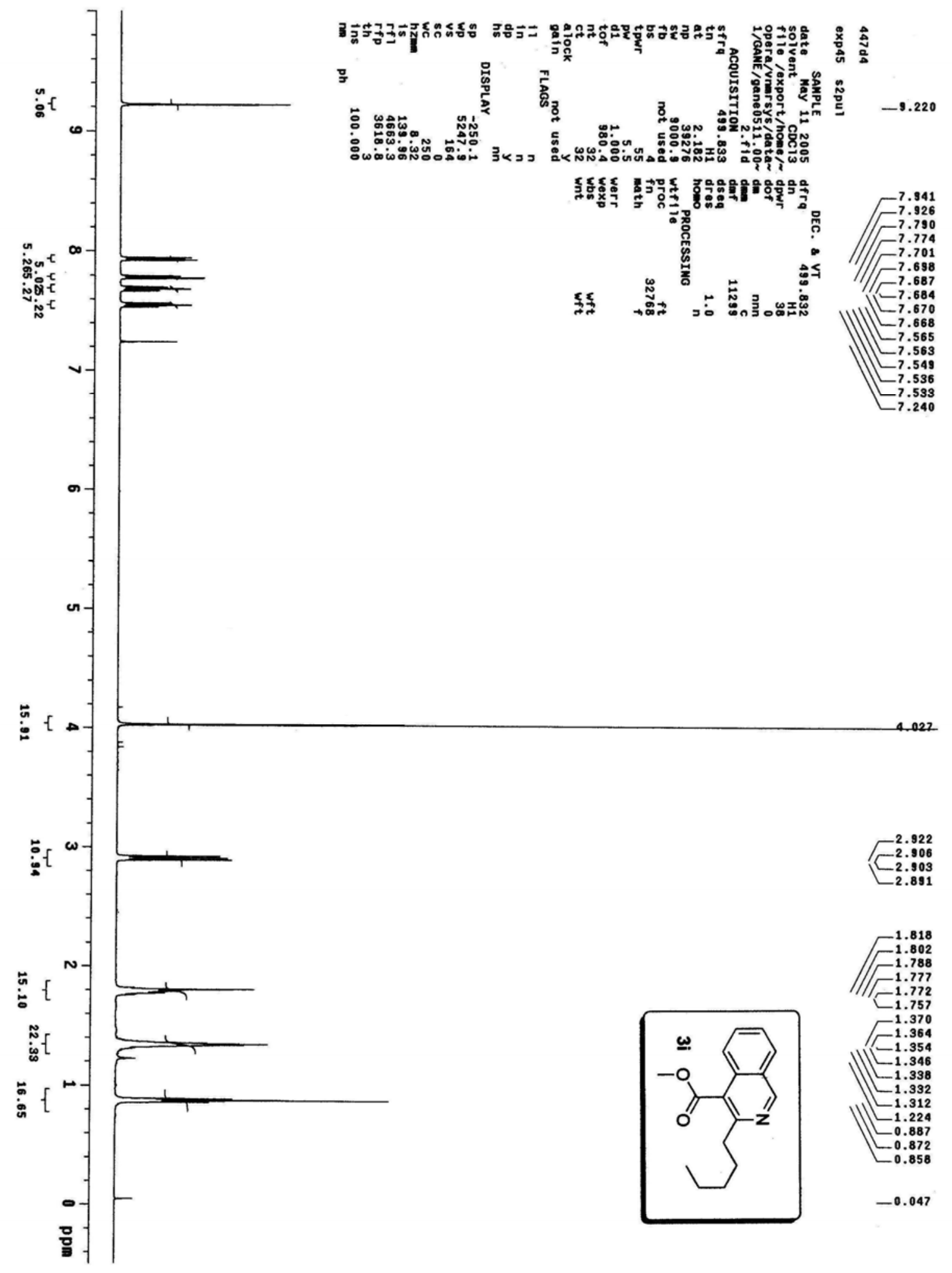




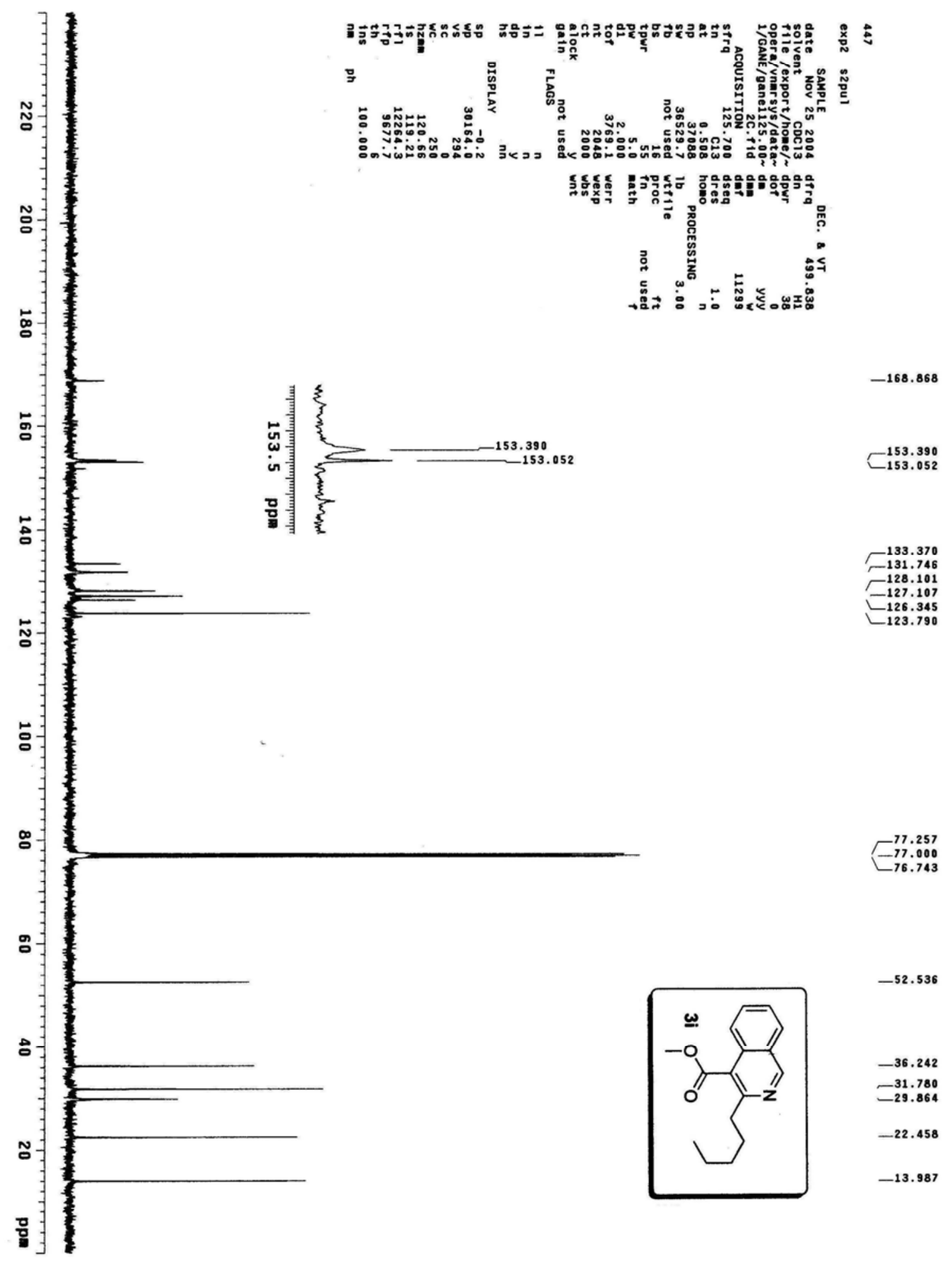




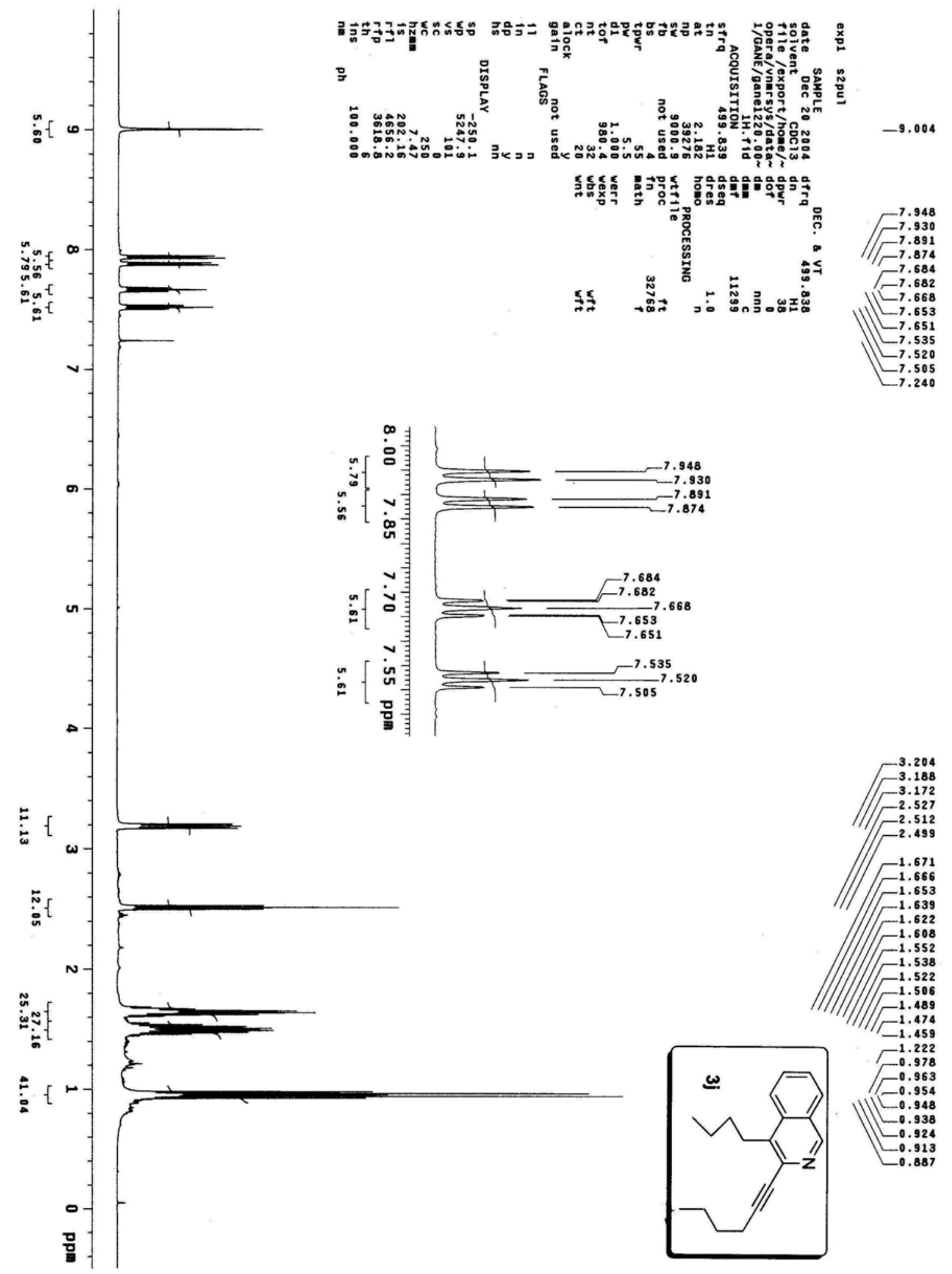



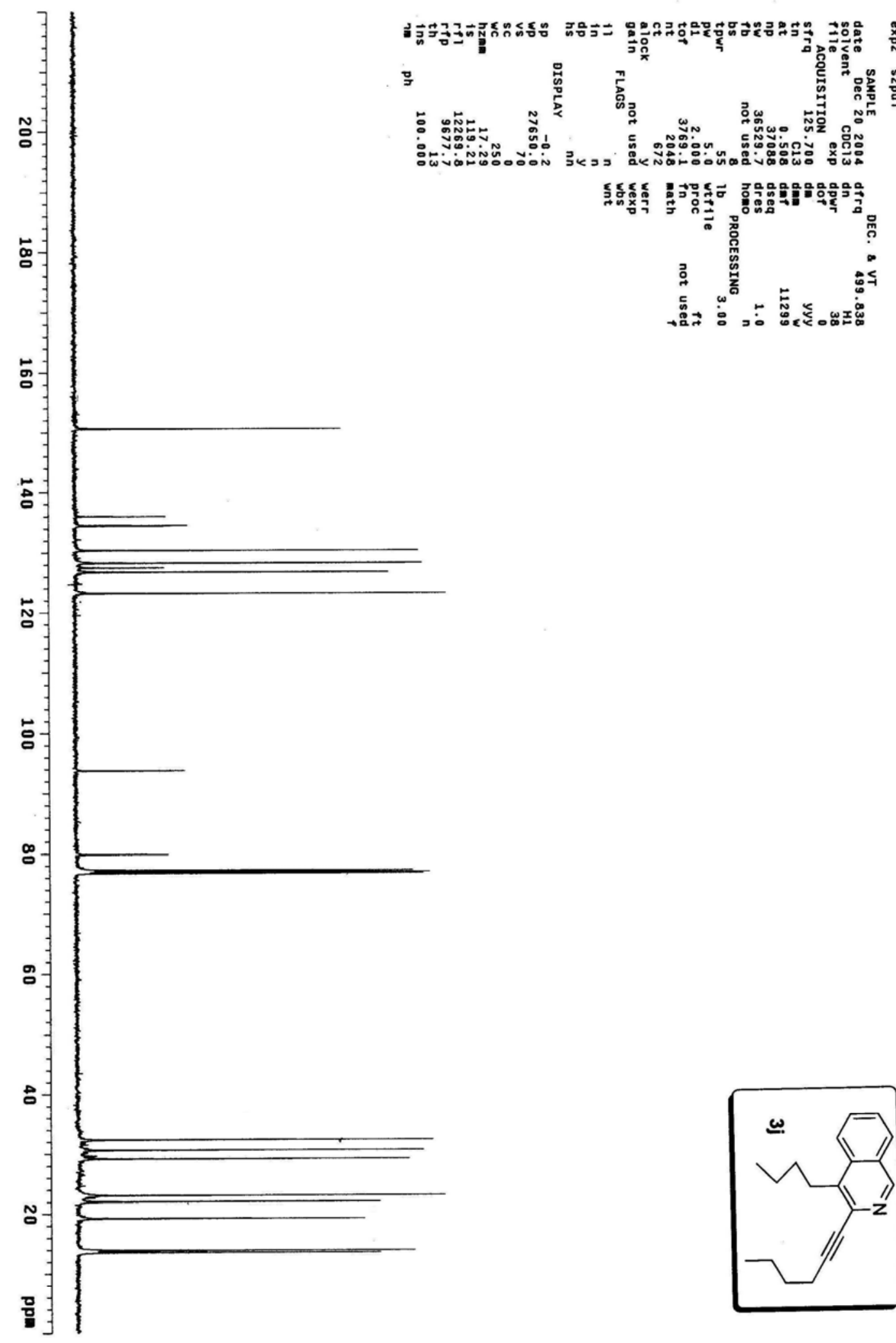

$-150.595$

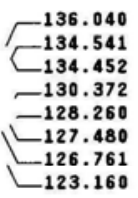

$-93.747$

$-79.865$ 77.257
-77.000 $-76.743$

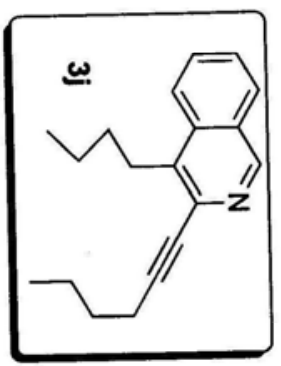

$-32.339$

$-30.609$

$-29.146$

-23.043
-22.014
-

$-19.255$

$-13.916$ 


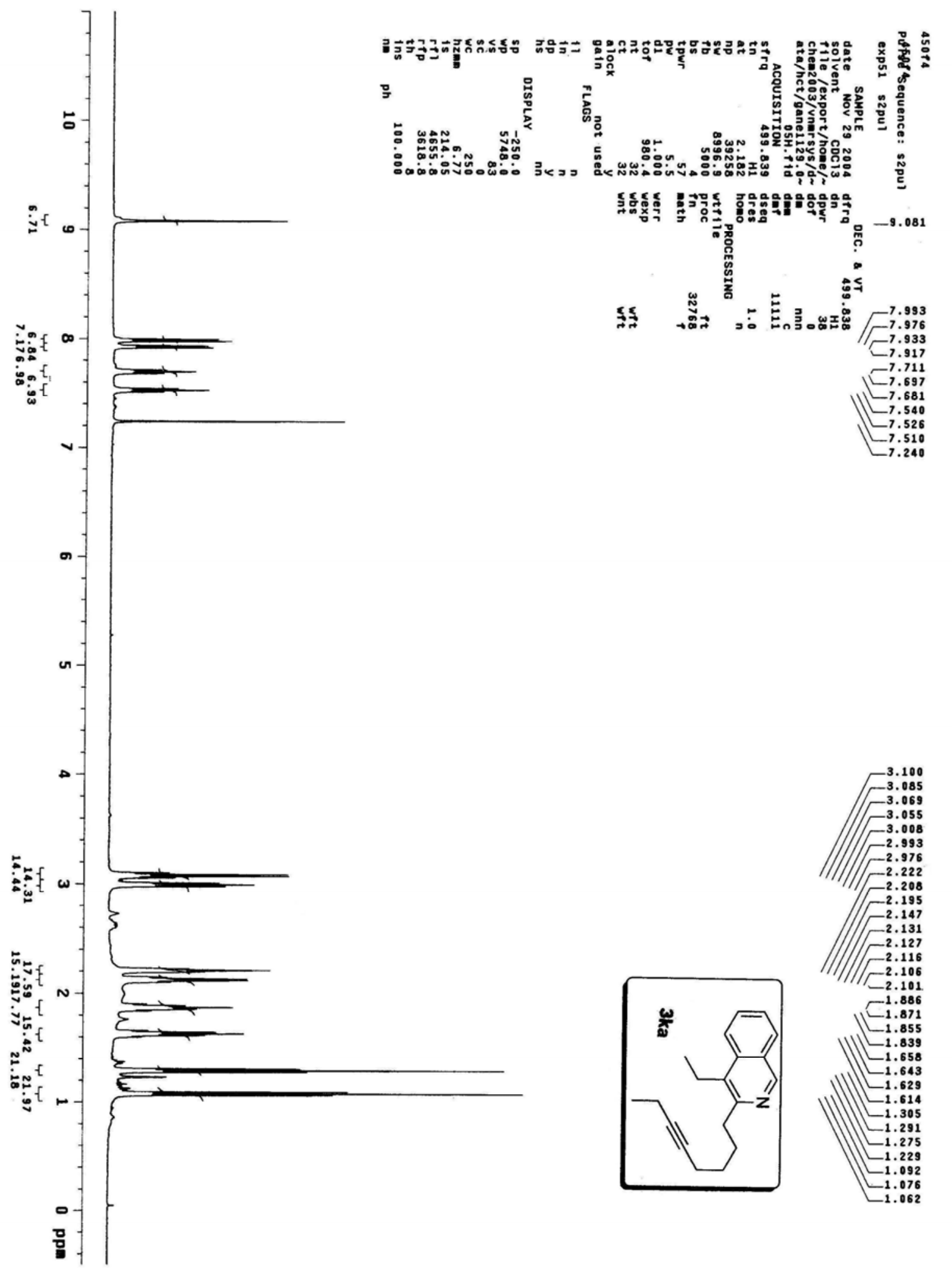




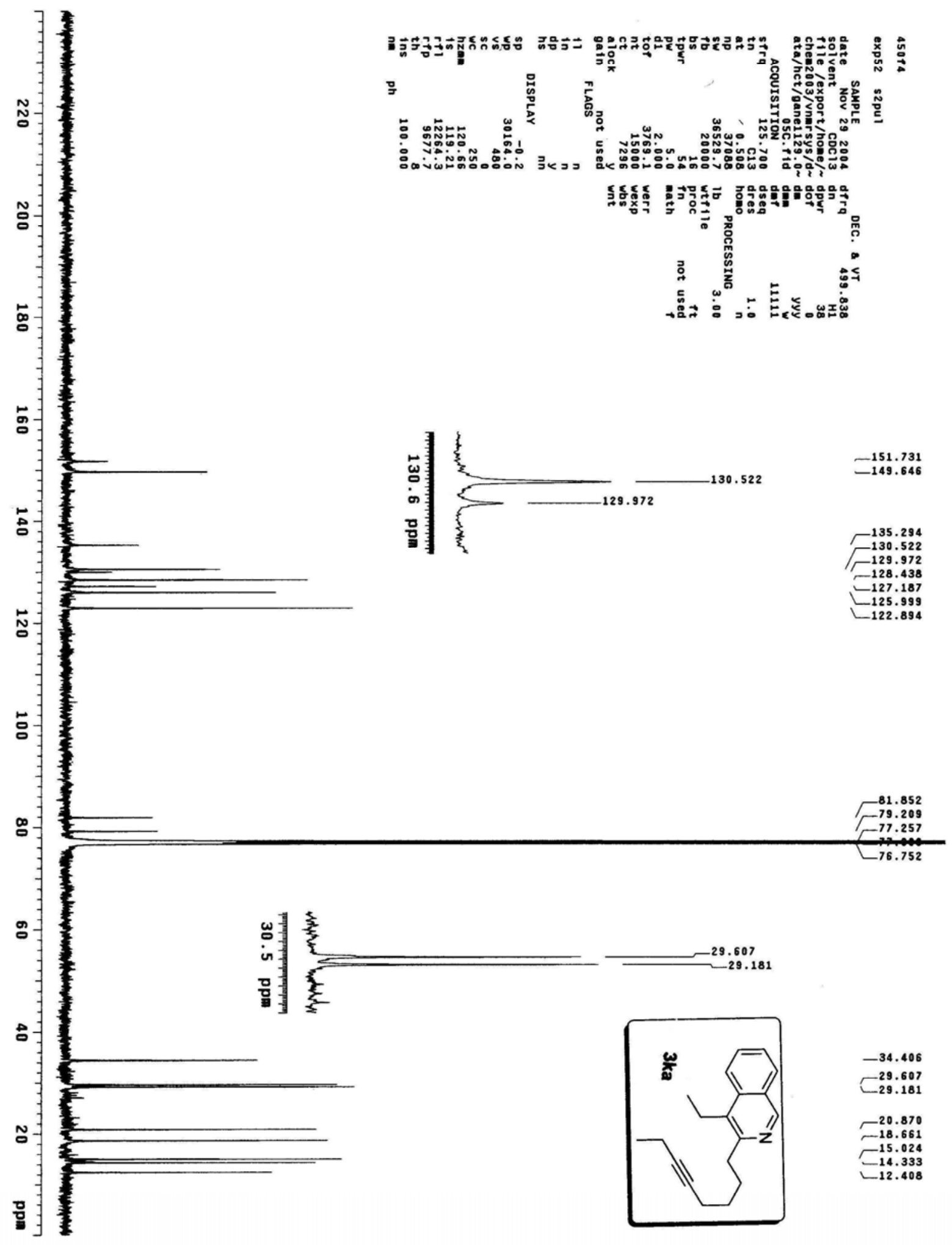



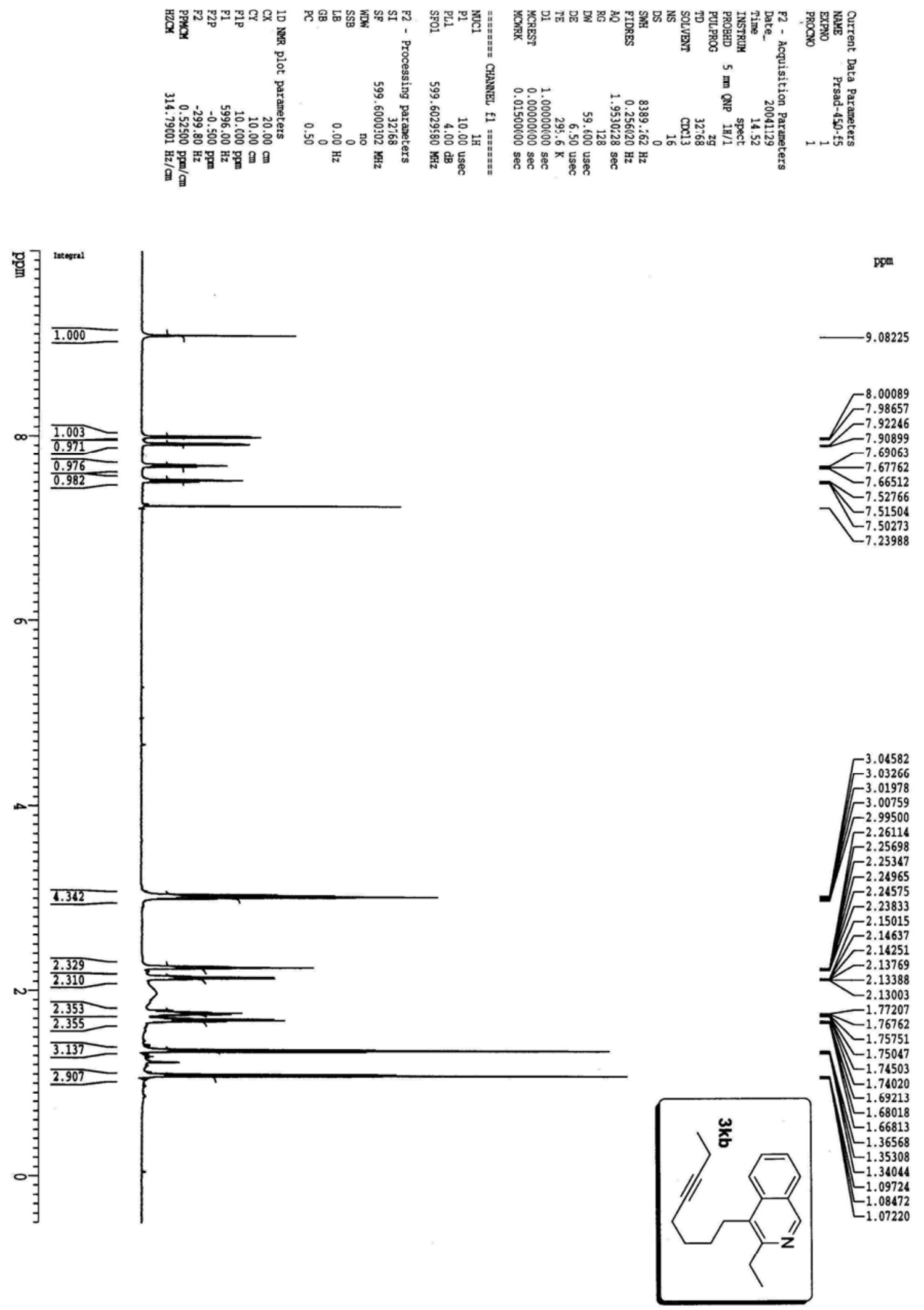

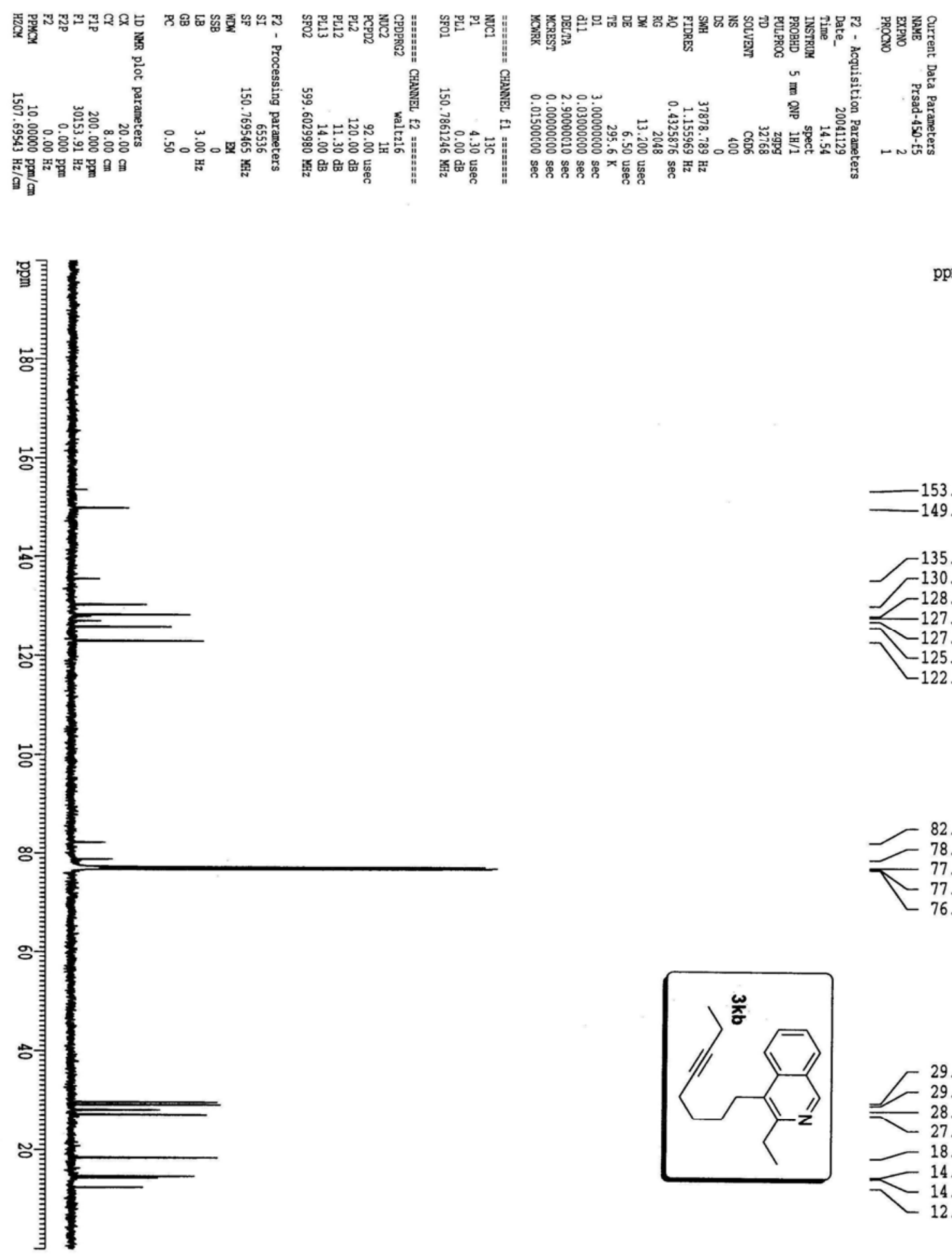

ppm

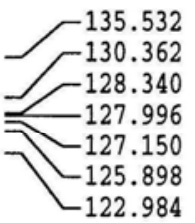

82.290

78.818

$-77.212$

$\sim 77.000$

76.788
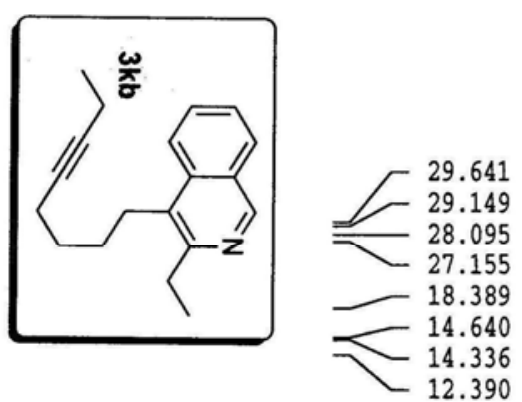


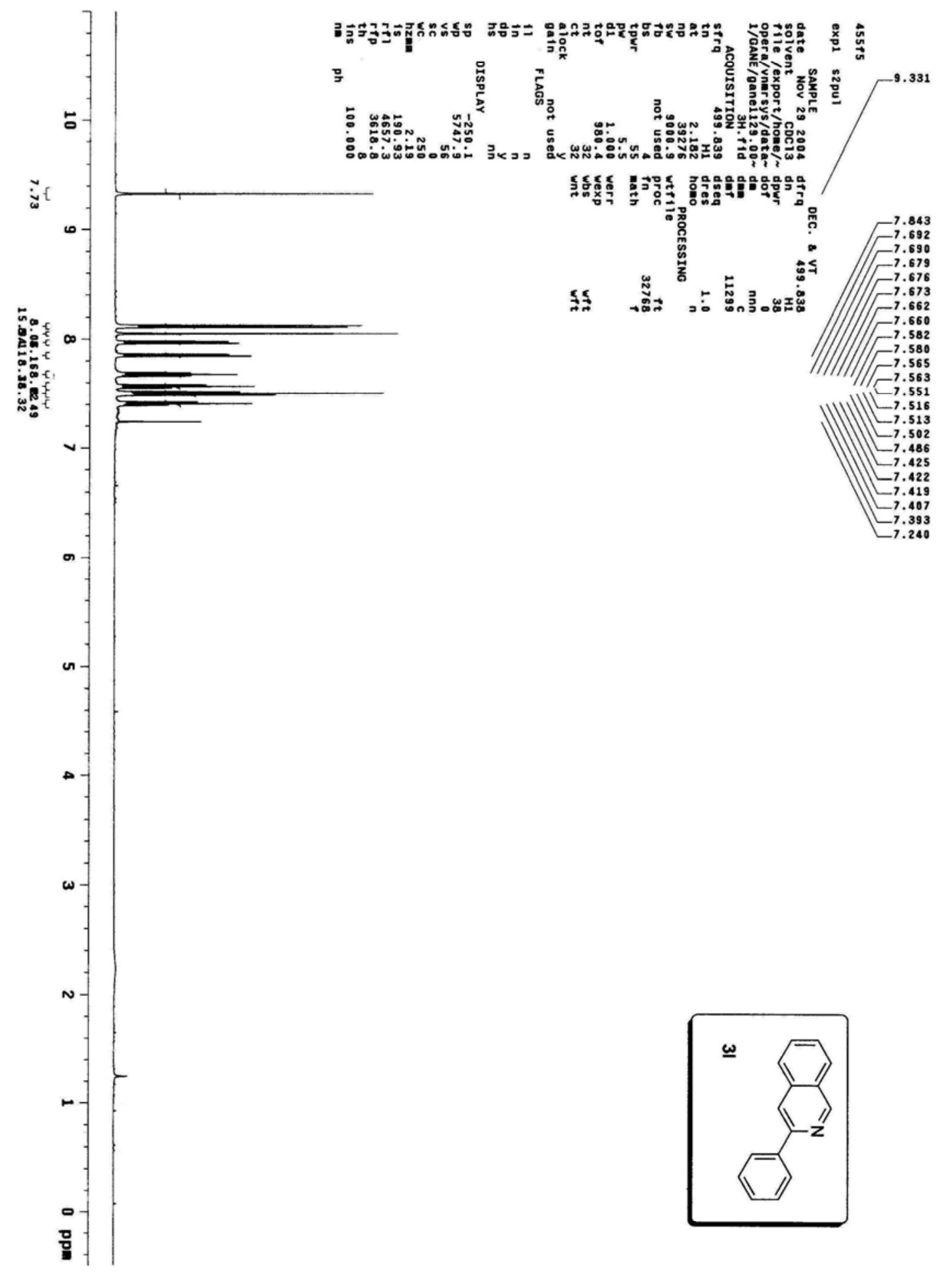




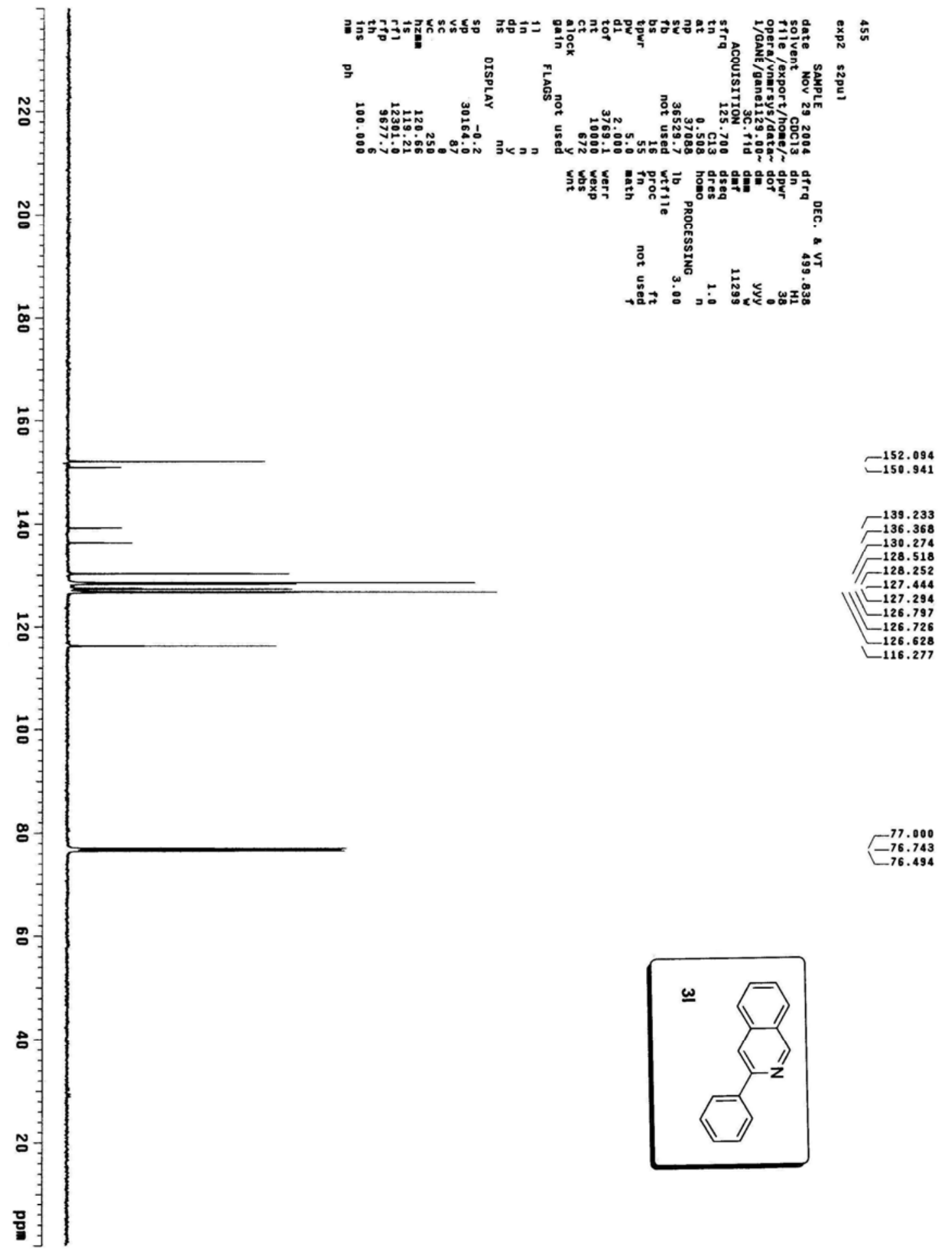




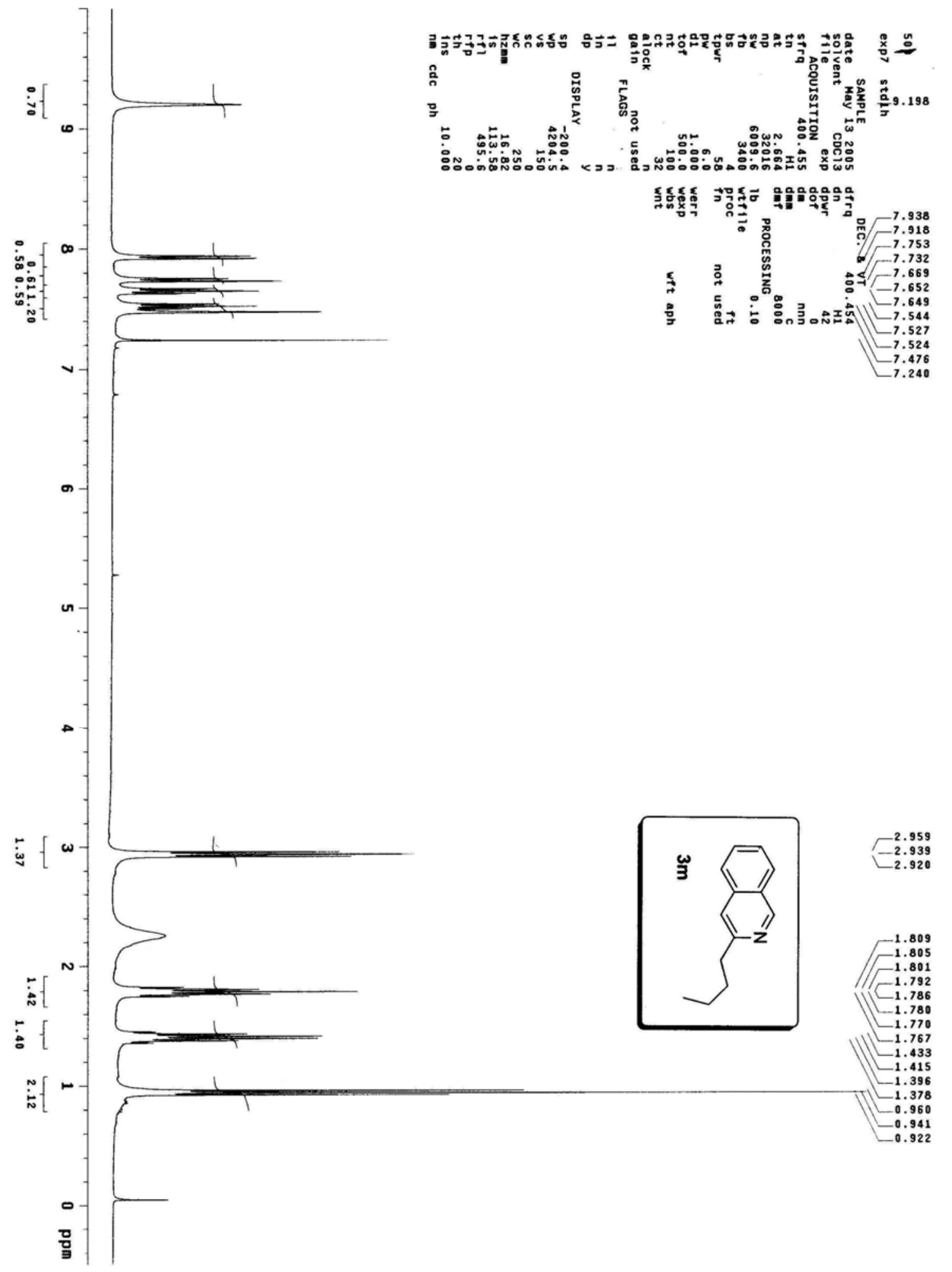




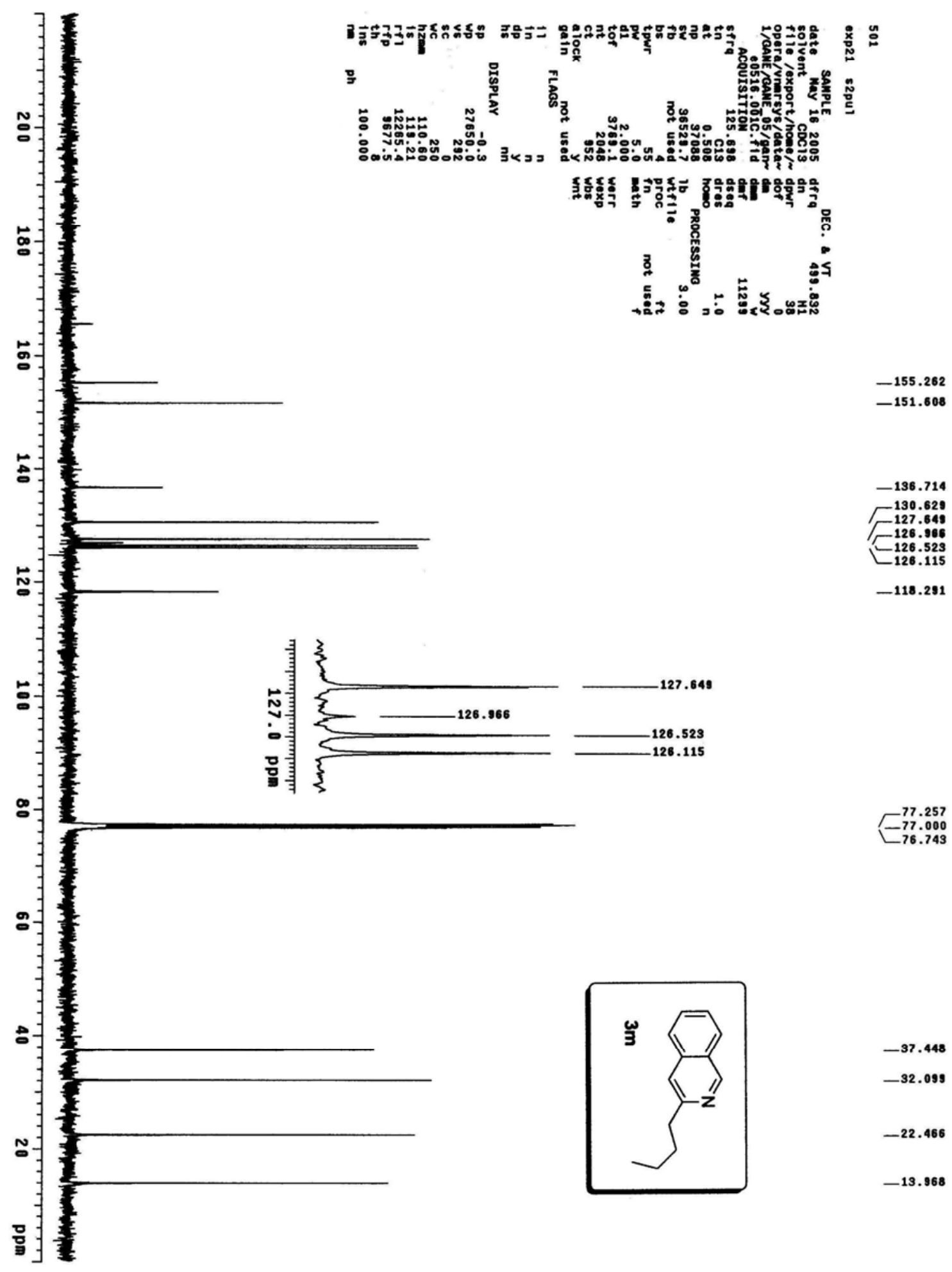




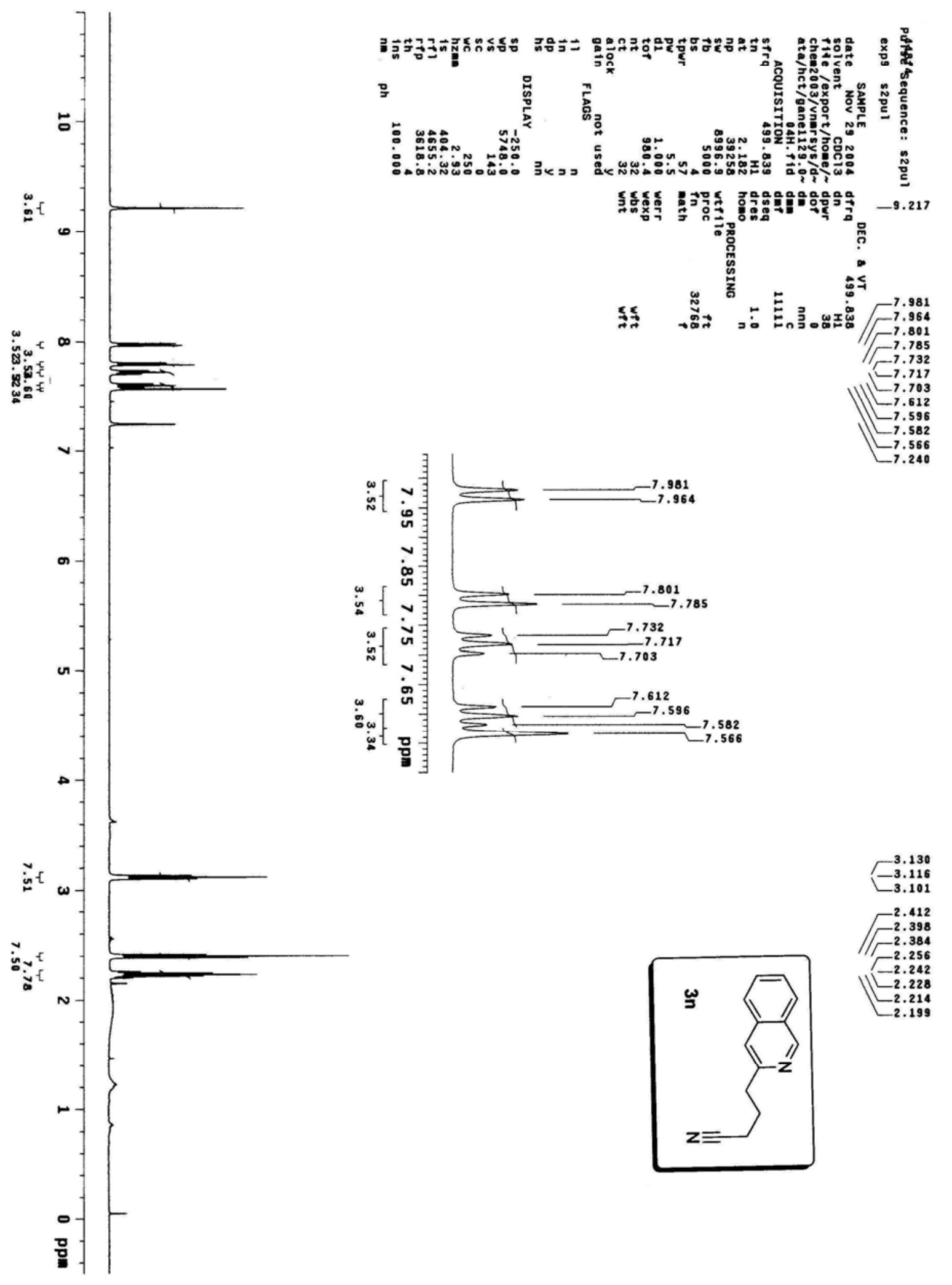




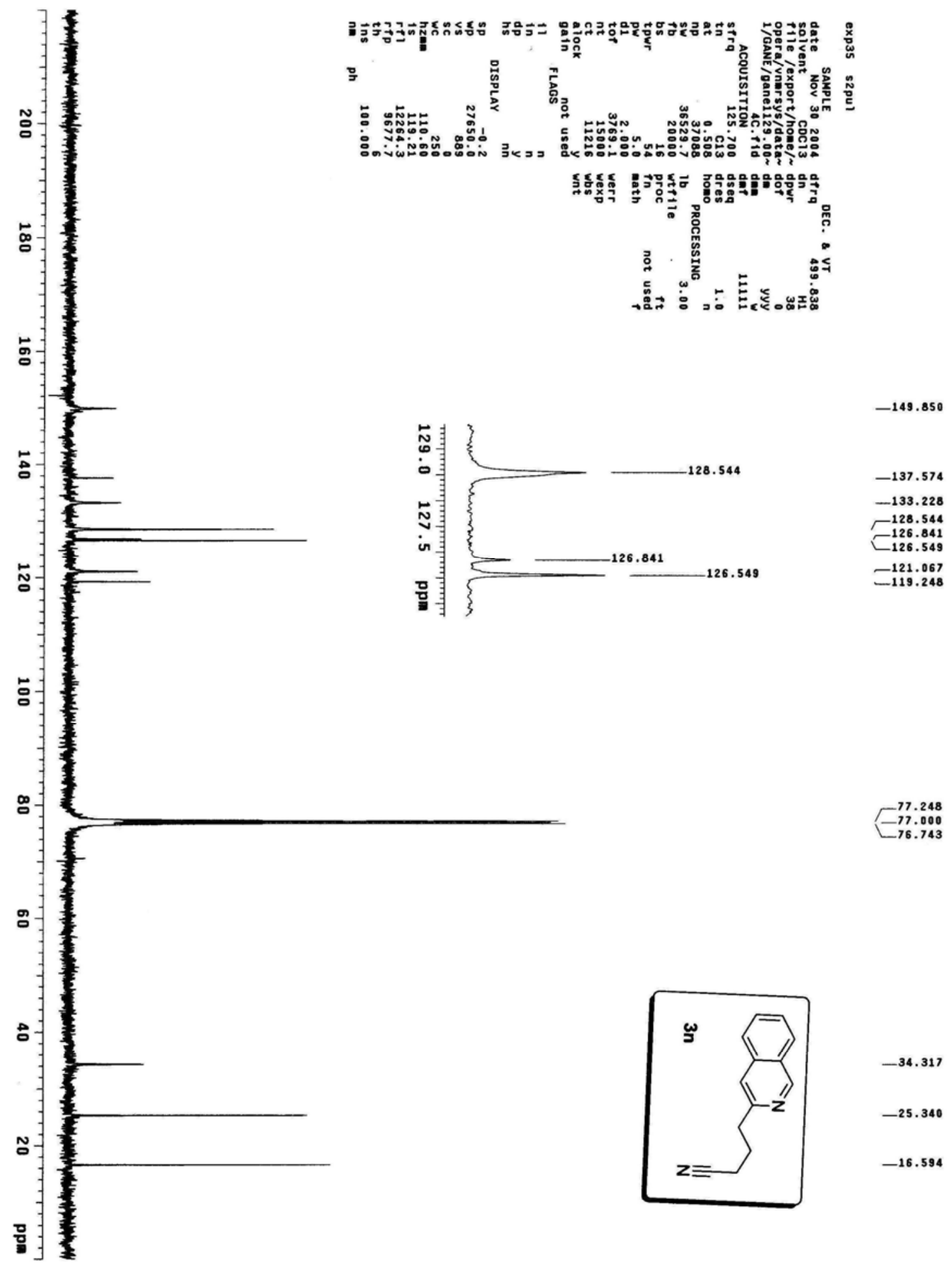




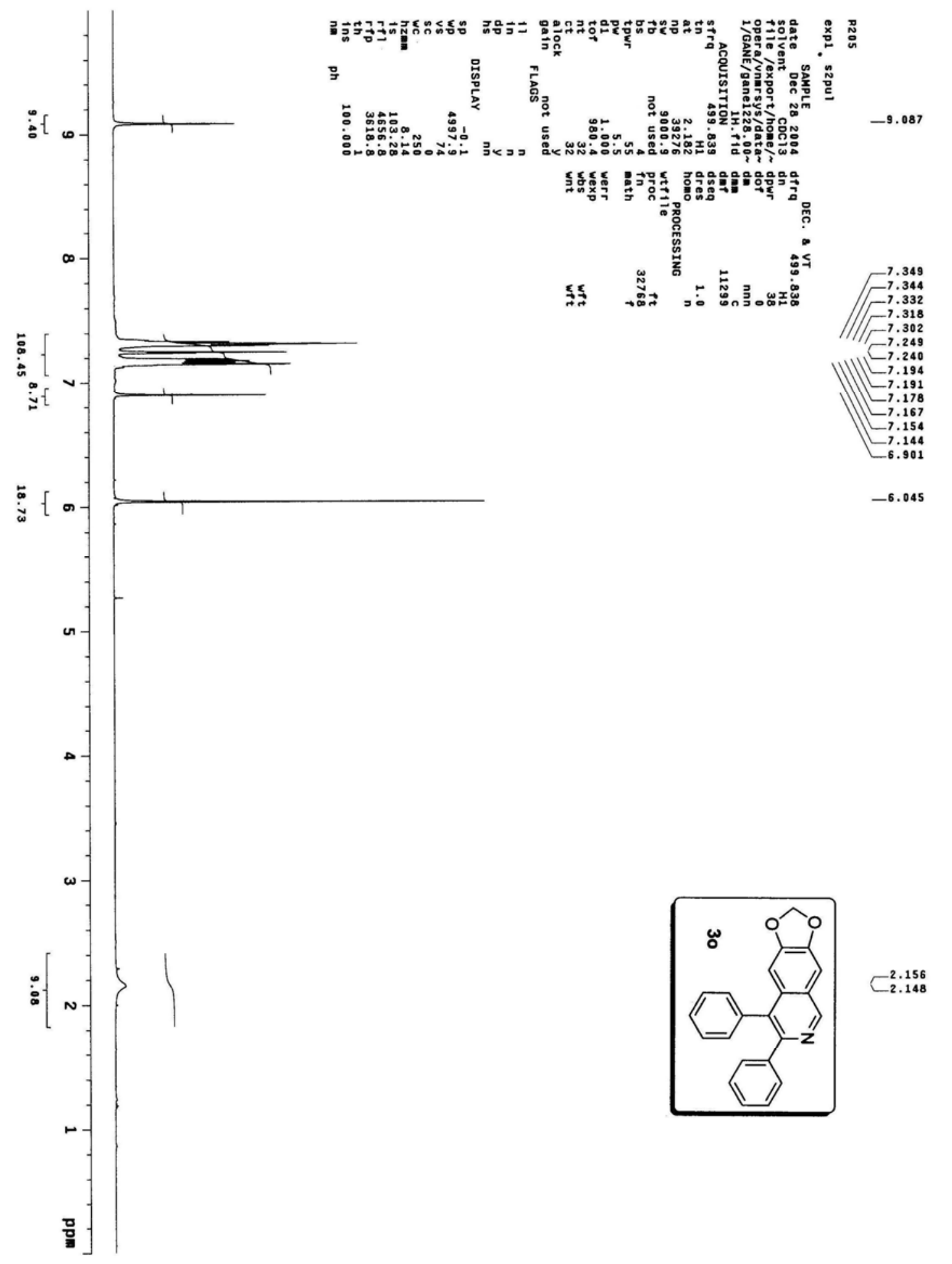




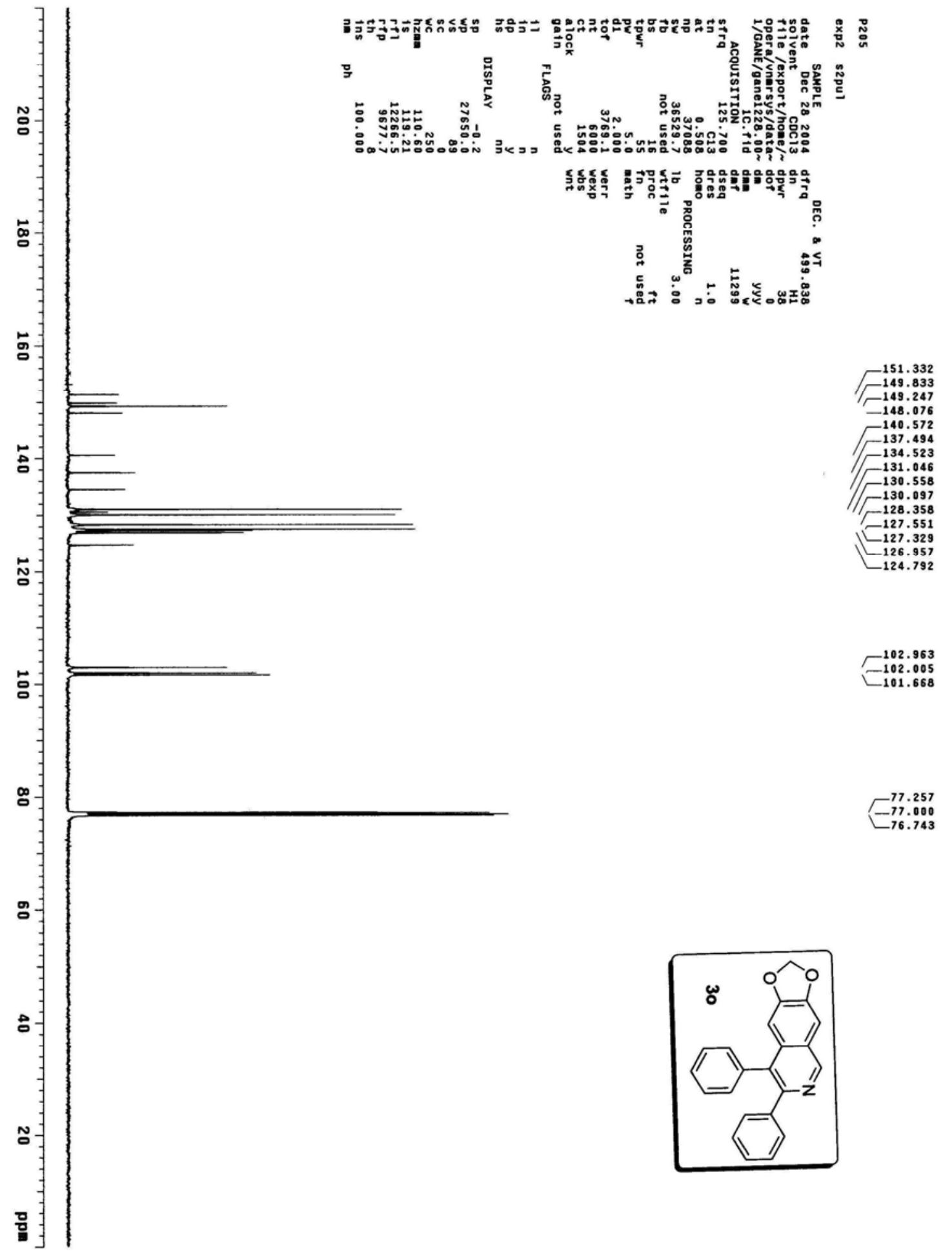



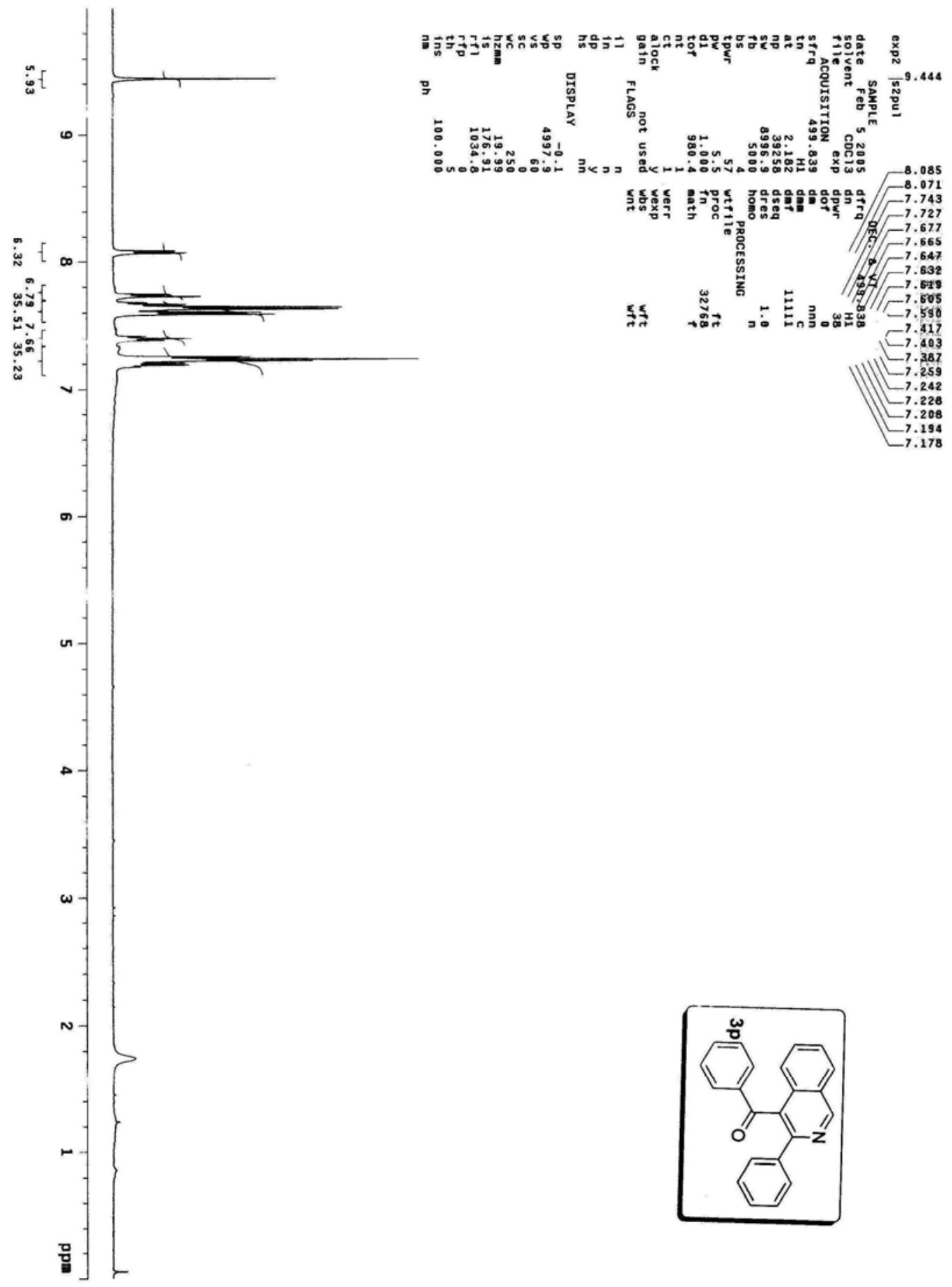

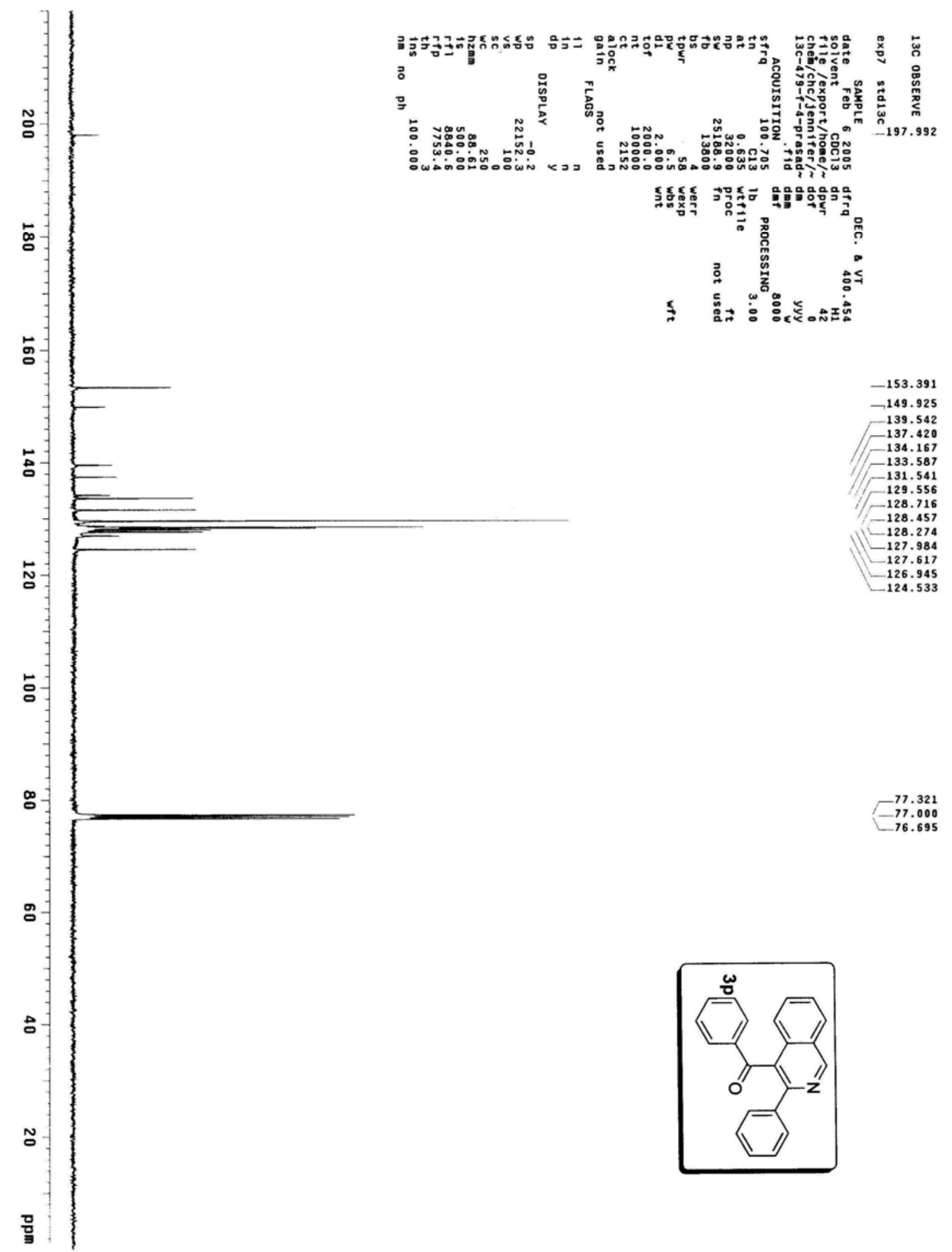


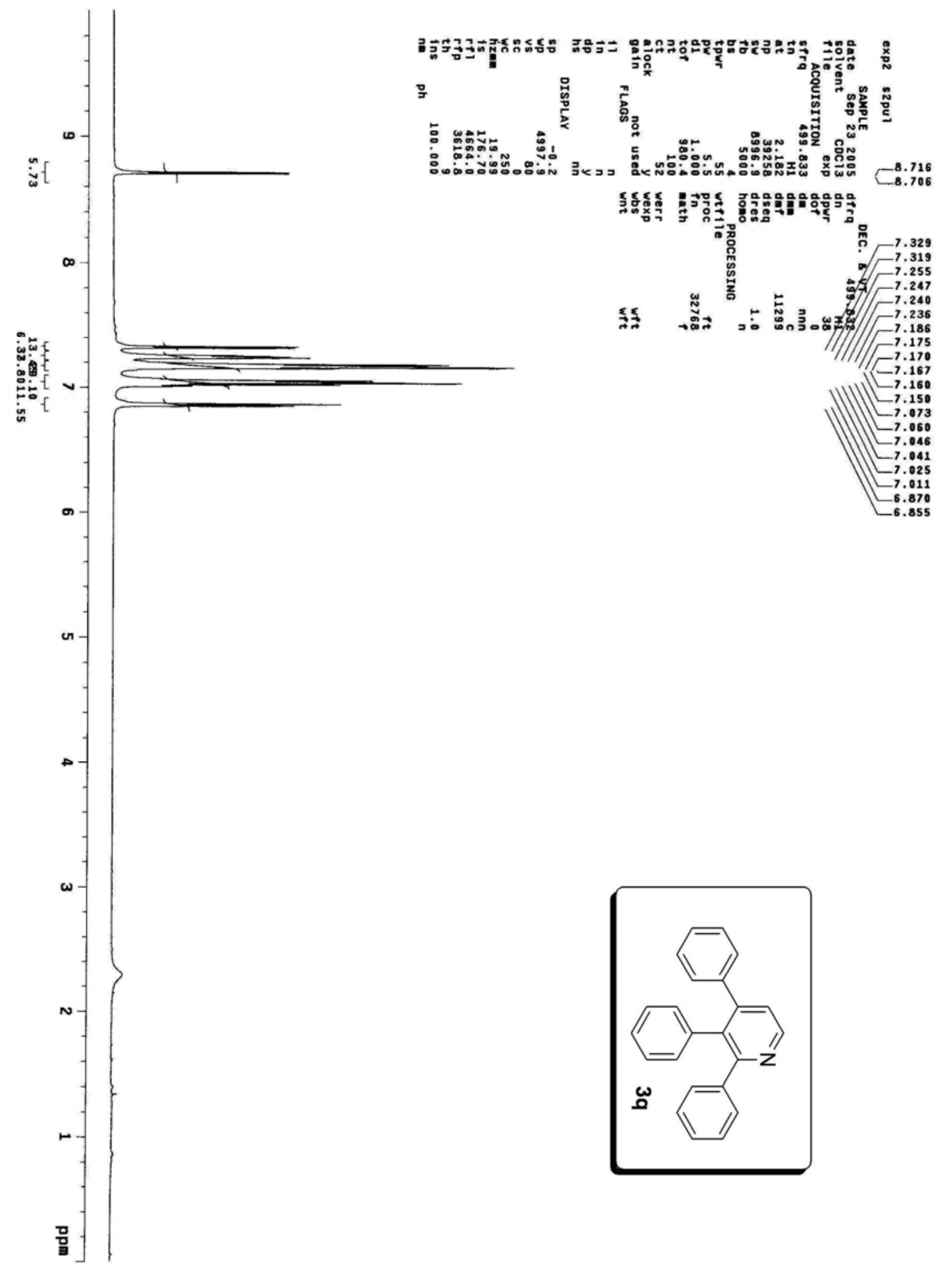




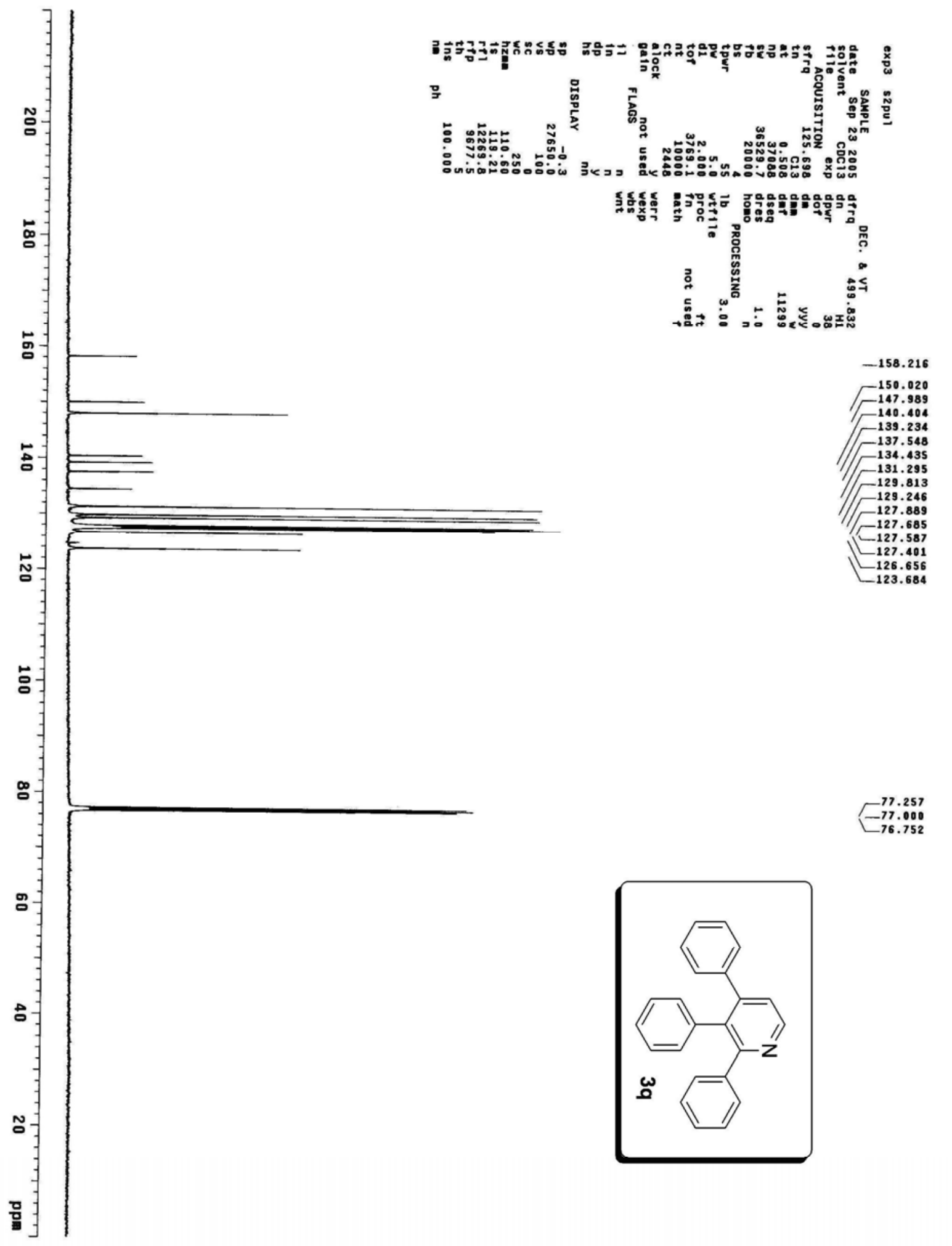

